\title{
Individual and Family Factors That Decrease Depression in Japanese, Chinese, and Korean Youth
}

\author{
Jennifer Ann Boeckel, PhD, LCSW \\ Department of Social Work, Minot State University \\ 1815 Schafer St. Office 152, Bismarck, North Dakota 58501 United States \\ E-mail: jennifer.boeckel@minotstateu.edu
}

Julie Anne Laser, PhD, LCSW

Graduate School of Social Work, University of Denver

2148 S High St, Denver, Colorado 80208, United States

Tel: 1-303-871-2352Ｅ-mail: julie.laser@du.edu

Received: January 24, 2015 Accepted: June 10, 2015 Published: August 7, 2015

doi:10.5296/ijsw.v2i2.6999 URL: http://dx.doi.org/10.5296/ijsw.v2i2.6999

\begin{abstract}
This study examined the risk and promotive factors that contribute to or decrease depression in late adolescents. Chinese, Japanese and Korean adolescents were surveyed in order to examine the individual and family factors influencing adolescents. Correlation and regression were used to examine the relationship among variables. Findings from the analysis revealed that promotive factors: autonomy, easy temperament, emotional intelligence, humor, moral development, optimism and physical beauty decreased depression. Risk factors that increased depression were history of physical abuse, history of sexual abuse, domestic violence, favoritism of siblings, parental depression and parent not aware. Implications of these findings for theory, research, policy and clinical practice are explored.
\end{abstract}

Keywords: Risk factors, Protective factors, Japanese youth, Chinese youth, Korean youth 


\section{Promotive and Risk Factors}

Promotive and risk factors have been defined in a variety of ways. Some authors refer to variables as if they are either uniquely promotive or uniquely risk factors (Ferguson \& Lynskey, 1996; Luthar, Cicchetti, \& Becker, 2000; Pollard, Hawkins, \& Athur, 1999; Rae-Grant, Thomas, Offord, \& Boyle, 1989). Others have emphasized that the promotive and risk factors are merely opposite ends of the same variable whether a variable was called a promotive or risk factor depends on which end of the continuum was emphasized (Kandel et al., 1988; White, Moffitt, \& Silva, 1989). Additionally, promotive factors have been conceptualized as processes that interact with risk factors in reducing the probability of a negative outcome (Rutter, 1985, 1990; Rutter, Giller, \& Hagell, 1998). If risk factors out number promotive factors, vulnerability for the individual will most likely be the outcome (Laser \& Nicoterr, 2011). However, if promotive factors out number risk factors resilience will more likely be the outcome (Laser \& Nicoterra, 2011). There has been a large body of research from the United States, Europe, and Australia that have explored these risk factors (eg. Carlton et al., 2006; Cicchetti \& Lynch, 1993; Ferguson \& Lynskey, 1996; Luthar, Cicchetti, \& Becker, 2000; Masten \& Powell, 2003; White, Moffitt, \& Silva, 1989) little research has been done in Asia.

\subsection{Indivdual Promotive Factors}

Individual promotive factors are those factors that reside within the youth individually and support resilience. The individual promotive factors in this research are: autonomy, creation of a personal myth, easy temperament, emotional intelligence, humor, moral development, optimism, physical beauty, and self-efficacy.

\subsubsection{Autonomy}

As adolescents develop they are eventually able to see themselves as independent self-regulating beings (Feldman \& Rosenthal, 1991). The adolescent's ability to be defined as their own unique individual and not merely in relationship to family or peers is the crux of autonomy.

\subsubsection{Creation of a Personal Myth}

Proposed by McAdams (1993, 2001), a sense of self is created through a personal life story. One's life story is the process used by youth and adults to help make sense out of the past and present and the awaited future (McLean, 2005). A personal myth gives youth a sense of purpose as well as, aspirations for the future (Howard, Dryden, \& Johnson, 1999; Wang, Haertel, \& Walberg, 1994). This promotes youth's sense of self, and allows them to maintain a belief that they have control over their future even when they are faced with difficult situations (Werner, 1990).

\subsubsection{Easy Temperament}

Youth who are characterized as having an easy temperament are found to have significantly lower levels of behavior problems than those youth with a difficult temperament (Ruschena, Prior, Sanson, \& Smart, 2005; Smith \& Prior, 1995; Tschann, Kaiser, Chesner, Alkon, \& 
Boyce, 1996; Wyman et al., 1999), higher levels of social competence (Smith \& Prior; 1995; Werner \& Smith, 1982; Werner \& Smith, 1992) and higher levels of adaptive behavior at home (Hetherington, 1989).

\subsubsection{Emotional Intelligence}

More important than cognitive ability is the ability to understand one's own emotions (Goleman, 1995, 1998, 2001). Emotional intelligence includes: self-awareness, self-management, social awareness, and relational management (Goleman, 1995, 1998, 2001). Having higher emotional intelligence has been found to reduce internalizing behaviors in youth (Austin, Saklofske, \& Egan, 2005; Izard, Fine, Schultz, Mostow, Ackerman, \& Youngstrom, 2001; Mavroveli, Petrides, Rieffe, \& Bakker, 2007).

\subsubsection{Humor}

Overall, having a sense of humor has been found to buffer psychological stress and the negative effects it can have on a person (Bricker, 1980; Civikly, 1986; Landy \& Mettee, 1969; Nezu, Nezu, \& Blissett, 1988; Smith \& Powell, 1988). Being able to reduce stress and tension for oneself and others is an important factor for resilience. Humor can protect children and gain affection from others (Wang, Haertel, \& Walberg, 1994). Positive humor can also bolster youth's psychological well-being (Martin, 2001). Humor can not only facilitate social behavior, but is associated with the reduction of depression, loneliness, and increased self-esteem (Overholser, 1992).

\subsubsection{Moral Development}

Adolescents begin to make choices because they are the right thing to do and not because they are going to gain positive favor or avoid criticism from others (Laser \& Nicotera, 2011).

\subsubsection{Optimism}

Optimism is important for adolescents to have especially in difficult times (Gilhan \& Reivich, 2004). In studies that look at youth who have a strong sense of optimism versus those learned helpless youth (Dweck \& Licht, 1980; Fincham, Hakoda, \& Sanders, 1989; Martinek \& Griffith, 1993; Stipek \& Kowalski, 1989) those youth with optimism are able to focus on figuring how to overcome the challenges in their way. Youth who are optimistic see challenges as temporary setbacks, versus a permanent place (Martinek \& Mellison, 1997). Those youth who do not have hope do not have the drive to want to change their circumstances (Martinek \& Mellison, 1997). Critical to enduring difficult times in life is having optimism and hopefulness (Seligman, 2006). Gilhan and Reivich (2004). Optimism is also related to less depression and anxiety and better physical health, and possibly a longer life (Gillham \& Reivich, 2004).

\subsubsection{Physical Beauty}

By comparing one's self to the internal representations of culturally determined ideas and standards of what physical appearance should be and what is aesthetically appealing define physical beauty (Faust, 1983; Fisher, 1990; Rosenblum \& Lewis, 1999; Sorell \& Nowark, 
1981).Those who are better looking or are perceived as better looking do better. Physical beauty has been shown to be an important aspect of self-worth and mental health (Harter, 1990).

\subsubsection{Self Efficacy}

Bandura (1977) introduced self-efficacy as influenced by mastery experiences, vicarious experiences, social persuasion, and physical and emotional states. When youth possess self-efficacy they are more likely to achieve the desired outcome they are less likely to have depressive symptoms and feelings of helplessness (Laser \& Nicotera, 2011).

\subsection{Family Promotive Factors}

Family promotive factors are behaviors of the family that support resilience. The family promotive factors reviewed in this research are: maternal relationship, paternal relationship, parental relationship, and parental social support.

\subsubsection{Maternal Relationship}

The relationship youth have with their parents will in part effect whether children are happy or sad, confident or unsure, or outgoing or withdrawn (Golombok, 2000). Having a good relationship with family, especially mothers, has been predictive of better adjustment (Laser et al., 2007a; Masten \& Coatsworth, 1998; Roth \& Brooks-Gunn, 2000).

\subsubsection{Paternal Relationship}

Fathers are also central players in the family and in the development of children (Parke et al., 2005). It has been found that youth who have a relationship with their fathers experience greater well-being (Amoato \& Gilbreth, 1999). Amoato and Gilbreth (1999) found an association between youths increased contact with their fathers and increased academic success and decreased depression.

\subsubsection{Parental Relationship}

The strength of the parental relationship improves adolescent functioning (Golombok, 2000). Marriage can go wrong in a variety of ways, but it is likely that people in trouble marriages will criticize, blame, and become angry with each other (Gottman, 1979, 1994). Marital satisfaction can have a positive impact on youth (Golombok, 2000). It has been shown that youth who have experienced parental discord are more aggressive, disobedient, and harder to control than youth who grow up in a stable parental relationship (Cummings \& Davies, 1994; Emery, 1988; Masten, 2013).

\subsubsection{Parental Social Support}

Social support plays an integral role in promoting mental health across the life span (Kashani, Canfield, Borduin, Soltys, \& Reid 1994). High family social support has been associated with a decrease in behavior problems and psychological distress in adolescents (Barrera \& Li, 1996; Borduin \& Schaffer, 1998; Cauce, Mason, Gonzales, Hiraga, Liu, 1996; Garnefski \& Diekstra, 1996; Plotnik, 1993). Kashani et al. (1994) demonstrated that youth need to have a 
sense that they are protected and supported by their families' support system in order to avoid both internalizing and externalizing behavior problems. Social support for parents can act as a protective factor against depression in youth, and can increase well-being and resilience (Hankin, 2006; Piko, 1998; Piko, Kovacs \& Fitzpatrick, 2009).

\section{Risk Factors}

Research has uniformly shown that the larger accumulation of risk factors, the higher the probability of negative outcomes later in life (Fergusson, Horwood \& Lynskey, 1996; Rutter, 1979; Sameroff et al., 1998; Smith et al., 2000). However, risk does not affect all people in the same way, some risks will affect all the children in a family, while other risk factors will only affect certain children (Sameroff, 2000). Even when controlling for age and developmental stage, children are still not equally affect by identical risk (Gore \& Eckenrode, 1996; Sameroff, 2000).

\subsection{Individual Risk Factors}

Individual risk factors are those factors that affect the youth individually potentially compromising resilience. The individual risk factors reviewed in this research are: history of physical abuse and history of sexual abuse.

\subsubsection{History of Physical Abuse}

Potential consequences of physical abuse have been identified as perceptual motor deficits, lower scores on measures of general intellectual functioning and academic achievement, negative social behaviors, aggressive behaviors, and lower self-worth (Ammerman, Cassisi, Hersen, \& Van Hasselt, 1986; Conaway \& Hansen, 1989; Fantuzzo, 1990; Lamphear, 1985; Laser et al., 2007a; Parker \& Herra, 1996). Youth who experience physical abuse are associated with self-injurious behaviors and suicidal behavior (Malinosky-Rummell \& Hansen, 1993). These youth also experience emotional problems such as somatization, anxiety, depression, dissociation, and psychosis (Malinosky-Rummell \& Hansen, 1993).

\subsubsection{History of Sexual Abuse}

Adolescents who have a history of sexual abuse experience higher rates of depression and dysthymia (Asarnow et al., 2011; Shamseddeen et. al., 2011) and posttraumatic distress disorder (Feiring \& Taska, 2005). Youth who experience sexual abuse experience low self-esteem as well as suicidal ideation or behavior (Brooks, 1985; Burgess, Hartman, McCausland, \& Powers, 1984; Gomes-Schwartz, Horowitz, \& Sauzier, 1985; Laser et al., 2007a; Lindberg \& Distad, 1985; Parker \& Herra, 1996; Runtz \& Briere, 1986).

\subsection{Family Risk Factors}

Family risk factors are those factors that can put youth at greater risk for depression. The family risk factors reviewed in this research are: domestic violence, favoritism of siblings, parental depression and parents not aware of youth's behavior. 


\subsubsection{Domestic Violence}

The effects of domestic violence have been described as having significant and measureable negative effects on a youth's functioning (Edleson, 1999; Fantuzzo \& Lindquist, 1989; Fantuzzo \& Mohr, 1999; Margolin \& Gordis, 2000; Wolak \& Finkelhor, 1998) including emotional and behavioral functioning (Silvern et al., 1995), social competence (Balog, 1995), school achievement (Whitbeck \& Simons, 1990), cognitive functioning (Rossman, 1998), psychopathology (Buka, Stichick, Birdthistle, Earls, 2001; Wolfe, Crooks, Lee, McIntyre-Smith, \& Jaffe, 2003), and general health (Graham-Bermann \& Seng, 2005).

\subsubsection{Favoritism of Siblings}

Favoritism can be defined as the "real or preferential treatment of one or more of a parent's children at the expense of that parent's other children" (Salmon, Shackelford, Michalski, 2012, p. 357). Parental favoritism has negative consequences for youth's lives (Kowal \& Kramer, 1997; Scholte, Engels, de Kamp, Harakeh \& Overbeek, 2007; Shanahan, McHale, Crouter \& Osgood, 2008). The disfavored child in the family is associated with poorer psychological well-being, whereas being the favored child is associated with greater well-being (Suitor, Sechrist, Plikuhn, Pardo \& Pillemer, 2008). Differential parental treatment in adolescence can increase externalizing behaviors such as aggression and acting out, and internalizing behaviors including depression, anxiety, and poor adjustment (Deater-Deckard, 2000; Dunn, Stocker, \& Plomin, 1990; Feinberg \& Hetherington, 2001; Harris \& Howard, 1984; McHale \& Gamble, 1989; McHale \& Paletko, 1992).

\subsubsection{Parental Depression}

Child abuse is found to be more likely to be present in families with depressed mothers than families where mothers are not depressed (Chaffin, Kekkeher, \& Hollenberg, 1996; Dixon, Hamilton-Giachritsis, \& Browne, 2005a; Dixon, Hamilton-Giachritsis, \& Browne, 2005b; Famularo, Kinscherff, \& Fenton, 1992; Kinard, 1996, Kotch, Browne, Dufort, Winsor, \& Catellier, 1999; Leschied, Chiodo, Whitehead, \& Hurley, 2005; Mullick, et. al. 2001; Sheppard, 1997; \& Walsh, MacMillan, Jamieson, 2003). Whether it is biological or environmental depressed parents are less likely to respond positively to their children. Parents who experience depression are more likely to have negative interactions with their children from infancy through adolescence (Field 1984; Jacob \& Johnson, 1997). Children of parents who are depressed can cause deleterious effects on parenting behaviors (Lovejoy et al., 2000) resulting in increased risk for externalizing behaviors (Ge et al., 1996; Heller \& Baker, 2000; Kim-Cohen et al, 2005) and internalizing behaviors (Wickramarante \& Weissman, 1998).

\subsubsection{Parents not aware of youth's Behavior}

Parental monitoring plays an important role in adolescent risk, and includes both supervision of youth and communication with youth (Stanton et al., 2000). Parental monitoring functions both as a primary preventive measure, reducing and slowing the onset of risk behavior, and as an intervention measure, reducing current risk behaviors (Stanton et al., 2000). 


\subsection{Internalizing Behaviors}

Behaviors that are directed inwardly towards the individual and represent an over controlled and inner-directed pattern of behavior are considered to be internalizing behaviors (Gresham \& Kern, 2004). Internalizing behaviors are subtle and can go unnoticed by others in the youth's world including parents (Gresham \& Kern, 2004). Internalizing behaviors can have a negative influence on youth's academic performance, physical health, future psychological adjustment, and even future employment opportunities (Flook, Repetti, \& Ullman, 2005; Merell, 1994; Merrell \& Walker, 2004). Depressed youth can lack the strategies used by other's to alleviate negative affects like problem solving or cognitive restructuring (Dodge \& Garber, 1991).

\section{Gender}

Many researchers have found girls to be more resilient than boys (Emery \& Forehand, 1996; Kumpfer, 1999; Wang, Haertel \& Walberg, 1994; Werner, 1994, 1985). Similarly, Rutter (1987) and Garcia-Coll, Lamberty, Jenkins, McAdoo, Crnic, Wasik and Garcia (1996) discuss that males are often at greater risk. Rutter (1987) believes that families protect female offspring by less punishment directed at females, less exposure to family discord, and closer monitoring. Interestingly, Werner (1994) finds that boys who were resilient were often the first-born. In contrast, Sameroff (2000) and Fergusson and Horwood (1999) found no difference between males and females regarding resilience. Splitting the sample by gender was important to better see the influence of the protective and risk factors. In some instance, only when the outcome variables were run by gender were the associations visible.

Thus four research questions were investigated:

1) What individual factors that have been shown to be important to the development of North American, European, and Australian youth impact the outcome of depression in Chinese, Korean and Japanese youth?

2) What family factors that have been shown to be important to the development of North American, European, and Australian youth impact the outcome of depression in Chinese, Korean and Japanese youth?

3) Are there differences in individual factors that impact the outcomes of internalizing behavior by gender in Chinese, Korean and Japanese youth?

4) Are there differences in family factors that impact the outcomes of internalizing behavior by gender in Chinese, Korean and Japanese youth?

\section{Method}

\subsection{Instruments}

The base survey is the same in all three countries, however scholars, researchers and youth workers from each country reviewed and modified wording of each item ensuring that items were culturally appropriate and that meaning of the terms used would be understood by the surveyed youth (Laser et al., 2007a,b). All measures were translated into the native language by native speakers and then translated back in to English by another to ensure proper translation (Laser et al., 2007a,b). Translators were bilingual, and were familiar with the 


\section{Macrothink \\ International Journal of Social Work \\ ISSN 2332-7278 \\ 2015, Vol. 2, No. 2}

nuances of psychological language and youth culture of respective countries (Laser et al., 2007a,b).

\subsubsection{The LEPFY}

The Laser Ecological Protective Factors for Youth (LEPFY) was designed to assess possible promotive factors. There are approximately 151 items on the survey and responses were given on a 5-point Likert scale (Laser et al., 2007a,b). Questions regarding individual, family, and extrafamilial promotive factors that impact youth in their environment were asked (Laser et al., 2007a,b). Items were created by Laser based on her research and review of the literature on promotive factors that have been linked to positive individual outcomes for at-risk youth in North America, Europe, New Zealand, and Australia (Laser et al., 2007a,b). Items also included in the LEPFY survey are those that would be of particular concern to scholars, researchers and youth workers of each respective country.

\subsubsection{The LESY}

The Life Events Survey for Youth (LESY) was designed to measure potential risk factors. There are approximately 114 items on the survey and responses were given on a 5-point Likert scale (Laser et al., 2007a,b). The LESY combines items from Small's Teen Assessment Project (TAP) Survey (Small \& Luster, 1994) with items found in the National Longitudinal Survey of Youth 1997 (NLSY97; Center for Human Resource Research, 2002) and items created by Laser based on her research and her investigation into the risk literature in North American, Europe, New Zealand, and Australia (Laser et al., 2007a,b). Items also included in the LESY survey are those that would be of particular concern to the scholars, researchers and youth workers of each respective country.

\subsubsection{Demographic Information Questionnaire}

Basic demographic questions regarding age, gender, and family composition of the respondent were asked on this survey (Laser et al., 2007a,b). The respondents were asked about their parent's academic background (Laser et al., 2007a,b) and other demographic characteristics the scholars, researchers and youth workers from each country thought were pertinent to know.

\subsection{Subscales}

All items were scored on a 5-point Likert scale. The reliability of the scale scores were estimated using Cronbach's alpha coefficients (Cronbach, 1951), all scales were found to be reliable (Table 1). 


MlMacrothink

Table 1. Initial scale reliabilities

\begin{tabular}{|c|c|c|}
\hline Measure & Number of Items & Alpha \\
\hline \multicolumn{3}{|l|}{ Individual Promotive Factors } \\
\hline Autonomy & 3 & .701 \\
\hline Creation of a Personal Myth & 3 & .860 \\
\hline Easy Temperament & 4 & .679 \\
\hline Emotional Intelligence & 4 & .770 \\
\hline Humor & 3 & .705 \\
\hline Moral Development & 4 & .740 \\
\hline Optimism & 3 & .726 \\
\hline Physical Beauty & 3 & .740 \\
\hline Self-efficacy & 3 & .792 \\
\hline \multicolumn{3}{|l|}{ Family Promotive Factors } \\
\hline Maternal Relationship & 3 & .812 \\
\hline Paternal Relationship & 3 & .808 \\
\hline Parental Relationship & 3 & .647 \\
\hline Parental Social Support & 4 & .827 \\
\hline \multicolumn{3}{|l|}{ Individual Risk Factors } \\
\hline History of Physical Abuse & 4 & .808 \\
\hline History of Sexual Abuse & 3 & .941 \\
\hline \multicolumn{3}{|l|}{ Family Risk Factors } \\
\hline Domestic Violence & 4 & .900 \\
\hline Favoritism of Siblings & 2 & .919 \\
\hline Parental Depression & 4 & .830 \\
\hline Parents Not Aware & 2 & .827 \\
\hline \multicolumn{3}{|l|}{ Negative Youth Outcomes } \\
\hline Delinquency & 4 & .905 \\
\hline Internalizing Behavior & 9 & .850 \\
\hline
\end{tabular}

\subsection{Sample}

The target population for this research was youth who were attending postsecondary institutions. In order to gather the data lengthy discussions with school officials took place before researchers entered into conversations with faculty and students. The total sample size 


\section{Macrothink}

of this research was 2539. Of that 1292 of the participants were Chinese from the Beijing area, 799 of the participants were Japanese from the Sapporo area, and 448 of the participants were Korean from the Seoul area. The total sample of participants' gender was $50.2 \%$ male and $48.7 \%$ female. The age of those surveyed was from 18-24 years old. The majority of the participants were between the ages of 18 and 20 years of age. The largest group of participants $(32.5 \%)$ was 19 years of age. The response rate of the anonymous survey was $99 \%$.

\section{Results}

\subsection{Bivariate Analysis}

Matrices were created to determine the bivariate relationships among the variables for the total sample (Figure 1), Chinese sample (Figure 2), Japanese sample (Figure 3), Korean sample (Figure 4). Many of the variables were related to each other, this is due to the large sample size. The number of correlations increase when the sample is split into male and female subsamples. Still, small to moderate correlations were the majority.

Correlations Among Promotive, Risk, and Outcome Variables for the Full Sample

\begin{tabular}{|c|c|c|c|c|c|c|c|c|c|c|c|c|c|c|c|c|c|c|c|c|}
\hline & 1. & 2 & 3. & 4. & 5. & 6. & ?. & 8. & 9. & 10. & 11. & 12. & 13. & 14. & 15. & 16 . & 17. & 18. & 19. & 20. \\
\hline 1. Autonory & - & & & & & & & & & & & & & & & & & & & \\
\hline \begin{tabular}{|l} 
2. Creation of a \\
Personal Nyth
\end{tabular} & $398 * *$ & - & & & & & & & & & & & & & & & & & & \\
\hline \begin{tabular}{|l|}
3 Fassy \\
Temperament
\end{tabular} & $.197 * 4$ & $465^{* *}$ & - & & & & & & & & & & & & & & & & & \\
\hline \begin{tabular}{|l|} 
4. Emotional \\
Intelligence
\end{tabular} & $348^{4 *}$ & $.646^{* *}$ & $503^{* 4}$ & - & & & & & & & & & & & & & & & & \\
\hline 5. Humor & $299 * 4$ & $.637^{* 4}$ & $494 * 4$ & $.659 \%$ & - & & & & & & & & & & & & & & & \\
\hline \begin{tabular}{|l|} 
6. Noral \\
Development
\end{tabular} & $362^{* *}$ & $581^{* *}$ & $295^{* *}$ & $.627^{* *}$ & $541^{* *}$ & - & & & & & & & & & & & & & & \\
\hline 7. Optimism & $312^{* 4}$ & $.737^{* *}$ & $416^{* *}$ & $577^{* *}$ & $.633^{* *}$ & $489^{* *}$ & - & & & & & & & & & & & & & \\
\hline \begin{tabular}{|l|} 
8.Physical \\
Besuty
\end{tabular} & $266^{* *}$ & $478^{* *}$ & $308^{* 4}$ & $462^{* 4}$ & $570^{* 4}$ & $.467^{* 4}$ & $411^{* *}$ & - & & & & & & & & & & & & \\
\hline 9. Self-Efficacy & $369^{4 * 4}$ & $.709^{* 4}$ & $465^{* *}$ & $.699 * 4$ & $.708^{* 4}$ & $.657^{* 4}$ & $.630^{\circ * *}$ & $544^{* *}$ & - & & & & & & & & & & & \\
\hline $\begin{array}{l}\text { 10. Matemal } \\
\text { Relationship }\end{array}$ & $293^{* *}$ & $.662^{* *}$ & 392 & $561^{* *}$ & $593^{4 *}$ & $566^{\circ 4}$ & $536^{* *}$ & $422^{\circ}$ & $.607^{* *}$ & - & & & & & & & & & & \\
\hline $\begin{array}{l}\text { 11. Patemal } \\
\text { Relationship }\end{array}$ & $246^{* *}$ & $592^{* 4}$ & $338^{* 4}$ & $.492^{* *}$ & $509^{* 4}$ & $509 * 4$ & $.437^{* *}$ & $358^{* 4}$ & $522^{\circ * 4}$ & $.692^{* *}$ & - & & & & & & & & & \\
\hline \begin{tabular}{|l|}
12 . Parsental \\
Relationship
\end{tabular} & $215^{* *}$ & $474^{* *}$ & $320^{* 4}$ & $478^{* *}$ & $.457^{* *}$ & $434^{* *}$ & $412^{* *}$ & $337^{* 4}$ & 477 & $567^{* *}$ & $518^{* *}$ & - & & & & & & & & \\
\hline \begin{tabular}{|l|} 
13. Parental \\
Social Support
\end{tabular} & $.159 * 4$ & $358^{* *}$ & $306^{* 4}$ & $326^{44}$ & $399 * 4$ & $346^{* 4}$ & $342^{* 4}$ & $310^{4 *}$ & $368^{4 *}$ & $398^{44}$ & $313^{* 4}$ & $272^{* 4}$ & - & & & & & & & \\
\hline \begin{tabular}{|l} 
14. History of \\
Physical Abuse
\end{tabular} & $.197 * *$ & $380^{* *}$ & $299^{* *}$ & $310^{* *}$ & $.404^{* *}$ & $351^{* *}$ & $364^{* *}$ & $391^{* *}$ & $.400^{* *}$ & $381^{* 4}$ & $241^{* *}$ & $260^{* *}$ & $355^{* *}$ & - & & & & & & \\
\hline $\begin{array}{l}\text { 15. History of } \\
\text { Sexual Abuse }\end{array}$ & $.170^{* *}$ & $338^{* *}$ & $.186^{* *}$ & $232^{* *}$ & $372^{* *}$ & $348^{* 4}$ & $304^{* *}$ & $392^{* *}$ & $285^{* *}$ & $277^{4 *}$ & $.139 * 4$ & $202^{* *}$ & $367^{* * 4}$ & $526^{6 * 4}$ & - & & & & & \\
\hline $\begin{array}{l}\text { 16. Domestic } \\
\text { Violence }\end{array}$ & $.197 * *$ & $314^{* *}$ & $.171^{* *}$ & $252^{* *}$ & $340^{* 4}$ & $310^{40}$ & $321^{* 4}$ & $341^{* *}$ & $302^{* *}$ & $242^{* 4}$ & $.061^{* 4}$ & $.125^{* 4}$ & $328^{\circ 4}$ & $504^{* 4}$ & $.717^{* 4}$ & - & & & & \\
\hline \begin{tabular}{|l} 
17. Favoritism of \\
Siblings
\end{tabular} & $.179 * *$ & $290^{* *}$ & $.138^{* 4}$ & $.185^{* *}$ & $361^{* *}$ & $309^{* *}$ & $262^{* *}$ & $384^{* 4}$ & $265^{* *}$ & $262^{* *}$ & $.130^{* *}$ & $.175^{* *}$ & $349^{* *}$ & $568^{* *}$ & $.733^{* 4}$ & $.627^{* 4}$ & - & & & \\
\hline \begin{tabular}{|l|} 
18. Parental \\
Depression
\end{tabular} & $.098^{* *}$ & $.138^{* *}$ & -017 & $.095 * *$ & $.175^{* *}$ & $.166^{* *}$ & $.186^{* *}$ & $217^{* *}$ & $.120^{* * *}$ & .085 & -018 & .006 & $201^{* *}$ & $405^{* *}$ & $598^{* 4}$ & $519^{* * *}$ & $593^{* *}$ & - & & \\
\hline $\begin{array}{l}\text { 19. Parents Not } \\
\text { Aware }\end{array}$ & $244^{* *}$ & $264^{* *}$ & $.109^{* 4}$ & $238^{44}$ & $303^{4 *}$ & $268^{* 4}$ & $294^{* *}$ & $295^{* *}$ & $309^{4 *}$ & $234^{4 *}$ & $.124^{* *}$ & $.129^{* 4}$ & $264^{4 *}$ & $465^{* 4}$ & $518^{* 4}$ & $490^{\circ *}$ & $517^{44}$ & $484^{\circ *}$ & - & \\
\hline 20. Delinquency & $.153^{\circ *}$ & $280^{\circ *}$ & $.137^{* *}$ & $.163^{* 4}$ & $344^{* *}$ & $301^{* 4}$ & $235^{* *}$ & $356^{4 *}$ & $247^{* 4}$ & $201^{* 4}$ & $.081^{* *}$ & $.123^{* 4}$ & $337^{\circ 4}$ & $521^{* *}$ & $828^{* 4}$ & $.709 * 4$ & $.713^{* 4}$ & $573^{\circ *}$ & $487^{\circ *}$ & - \\
\hline $\begin{array}{l}\text { 21. Intemalizing } \\
\text { Behavior }\end{array}$ & $243^{* *}$ & $311^{* 4}$ & $.073^{4 *}$ & $249^{* *}$ & $349^{* *}$ & $301^{* *}$ & $395^{* *}$ & $368^{* 4}$ & $349^{4 *}$ & $332^{4 *}$ & $.169^{* 4}$ & $218^{4 *}$ & $348^{* *}$ & $.605^{4 *}$ & $.685^{* *}$ & $587^{* 4}$ & $.697^{4 *}$ & $.612^{44}$ & $.624^{* 4}$ & $.631^{* *}$ \\
\hline
\end{tabular}

Figure 1. Correlations among promotive, risk and outcome variable for the full sample 
Correlations Among Promotive, Risk, and Outcome Variables for the Full Chinese Sample

\begin{tabular}{|c|c|c|c|c|c|c|c|c|c|c|c|c|c|c|c|c|c|c|c|c|}
\hline & 1. & 2. & 3. & 4. & 5. & 6. & 7. & 8. & 9. & 10. & 11. & 12. & 13. & 14. & 15. & 16. & 17. & 18. & 19. & 20. \\
\hline 1. Autonony & - & & & & & & & & & & & & & & & & & & & \\
\hline $\begin{array}{l}\text { 2. Creation of a } \\
\text { Personal Nyth }\end{array}$ & $.063^{*}$ & - & & & & & & & & & & & & & & & & & & \\
\hline \begin{tabular}{|l|} 
3. Easy \\
Terpperament
\end{tabular} & -.006 & $204^{* *}$ & - & & & & & & & & & & & & & & & & & \\
\hline \begin{tabular}{|l|} 
4. Emotional \\
Intelligance
\end{tabular} & .022 & $297^{* *}$ & $278^{* *}$ & - & & & & & & & & & & & & & & & & \\
\hline 5. Humor & -.009 & $306^{* *}$ & $351^{* *}$ & $370^{* *}$ & - & & & & & & & & & & & & & & & \\
\hline \begin{tabular}{|l|} 
6. Moral \\
Development
\end{tabular} & .005 & $.157^{* *}$ & $-.065^{*}$ & $285^{* *}$ & $.162^{* *}$ & - & & & & & & & & & & & & & & \\
\hline 7. Optimism & .006 & $552^{* *}$ & $237^{* *}$ & $308^{* *}$ & $352^{* *}$ & $.105^{* *}$ & - & & & & & & & & & & & & & \\
\hline $\begin{array}{l}\text { 8. Physical } \\
\text { Beauty }\end{array}$ & $.085^{* *}$ & $.164^{* *}$ & $.127^{* *}$ & $.114^{*}$ & $.162^{*}$ & $.161^{* *}$ & .039 & - & & & & & & & & & & & & \\
\hline 9. Self-Efficacy & $.078^{* 4}$ & $460^{* *}$ & $223^{* *}$ & $389 * *$ & $.409^{* *}$ & $.443^{* *}$ & $217^{* *}$ & $.198^{* 4}$ & - & & & & & & & & & & & \\
\hline \begin{tabular}{|l|} 
10. Matemal \\
Relationship
\end{tabular} & $-.089 * 4$ & $297 * *$ & $.098^{* *}$ & $.166^{* *}$ & $218^{* *}$ & $221^{* *}$ & $.124^{* *}$ & .049 & $.170^{* *}$ & - & & & & & & & & & & \\
\hline \begin{tabular}{|l} 
11. Patemal \\
Relationship
\end{tabular} & $-094^{* 4}$ & $279 * *$ & $.103^{* *}$ & $.160^{* *}$ & $207 * *$ & $242^{* *}$ & $.098^{* *}$ & $107 * *$ & $.189^{4 *}$ & $525^{* 4}$ & - & & & & & & & & & \\
\hline \begin{tabular}{|l|} 
12. Parental \\
Relationship
\end{tabular} & $-.064^{*}$ & $.147^{* *}$ & .048 & $.141^{* *}$ & $.149 * *$ & $.134^{* *}$ & $.070^{\circ}$ & $.056^{*}$ & $.128^{* *}$ & $357^{4 *}$ & $2974 *$ & - & & & & & & & & \\
\hline $\begin{array}{l}\text { 13. Parental } \\
\text { Social Support }\end{array}$ & .051 & $.125^{* *}$ & $.127^{* *}$ & $.130^{* *}$ & $.161^{* *}$ & $.136^{* 4}$ & $.112^{* *}$ & .049 & $.096^{* *}$ & $.138^{* 4}$ & $.133^{* *}$ & $.158^{* *}$ & - & & & & & & & \\
\hline $\begin{array}{l}\text { 14. History of } \\
\text { Physical Abuse }\end{array}$ & -.010 & $-.070^{*}$ & -.021 & -.022 & -.020 & $-063^{*}$ & .008 & -.021 & .011 & $-056^{*}$ & $-.108^{* 4}$ & -.028 & .008 & - & & & & & & \\
\hline $\begin{array}{l}\text { 15. History of } \\
\text { Sexual Abuse }\end{array}$ & $.056^{*}$ & $-103^{* *}$ & -.024 & .046 & -.001 & $-.067^{*}$ & -033 & $116^{* *}$ & .064 & $-.072^{* *}$ & $-.078^{* *}$ & .000 & -.012 & .047 & - & & & & & \\
\hline \begin{tabular}{|l|} 
16. Domeatic \\
Violence
\end{tabular} & $.067^{*}$ & $-074^{* *}$ & $-.088 * 4$ & .030 & $-075^{* *}$ & $-.080^{* *}$ & $.073^{* *}$ & .032 & .009 & $-165^{* *}$ & $-300^{* *}$ & $-175^{* *}$ & $-073^{* 4}$ & $.143^{* *}$ & $.093^{* *}$ & - & & & & \\
\hline $\begin{array}{l}\text { 17. Favoritism of } \\
\text { Siblings }\end{array}$ & .049 & $-167^{* *}$ & $-.098^{*}$ & $-0922^{*}$ & $-092^{*}$ & $-.128^{* 4}$ & -.053 & .074 & $-140^{* *}$ & $-155^{\circ *}$ & $-131^{* *}$ & $-157^{* *}$ & -021 & $257^{* *}$ & $.181^{* *}$ & $.163^{* *}$ & - & & & \\
\hline \begin{tabular}{|l} 
18. Parental \\
Depression
\end{tabular} & .047 & $-155^{* *}$ & $-201^{* *}$ & $-.073^{* *}$ & $-102^{* *}$ & $-117^{* *}$ & .035 & .011 & $-117^{* *}$ & $-218^{* *}$ & $-247 * *$ & $-26 g^{* *}$ & .005 & $.158^{* *}$ & $.098^{* *}$ & $233^{* *}$ & $.190^{* *}$ & - & & \\
\hline $\begin{array}{l}\text { 19. Parentis Not } \\
\text { Aware }\end{array}$ & $.135^{\circ *}$ & $-.171^{* 4}$ & $-157^{* 4}$ & $-.064^{*}$ & $-.121^{* *}$ & $-.127 * *$ & .007 & .043 & -.051 & $-3333^{* 4}$ & $-292 * 4$ & $-273^{* 4}$ & $-.076^{* 4}$ & $.137^{* *}$ & .044 & $213^{* *}$ & $151^{* *}$ & $284^{* 4}$ & - & \\
\hline 20. Delinquency & $.062^{*}$ & $-055^{*}$ & -037 & $.062^{*}$ & $-055^{*}$ & -037 & $-.064^{*}$ & .033 & -019 & -018 & $-120^{* *}$ & $-112^{*}$ & -014 & $.188^{\circ *}$ & $.148^{* *}$ & $285^{* *}$ & $221^{* *}$ & $.156^{* *}$ & $.151^{* *}$ & - \\
\hline \begin{tabular}{|l} 
21. Intemalizing \\
Behavioo
\end{tabular} & $.095 * *$ & $-315^{* 4}$ & $-464^{* *}$ & $-224^{* 4}$ & $-280^{* * 4}$ & -29744 & $.079 * *$ & $-.057^{*}$ & $-1599^{* *}$ & $-208^{* 4}$ & $-274^{* *}$ & $-220^{* *}$ & $-0904 \%$ & $185^{* *}$ & $119 * *$ & $.190^{* *}$ & $226^{* *}$ & $349 * *$ & $365^{* *}$ & $.176^{* *}$ \\
\hline
\end{tabular}

Figure 2. Correlations among promotive, risk and outcome variable for the full Chinese sample 
Correlations Among Promotive, Risk, and Outcome Variables for the Full Japanese Sample

\begin{tabular}{|c|c|c|c|c|c|c|c|c|c|c|c|c|c|c|c|c|c|c|c|c|}
\hline & 1. & 2. & 3. & 4. & 5. & 6. & ?. & 8. & 9. & 10. & 11. & 12 . & 13. & 14. & 15 . & 16. & 17. & 18. & 19. & 20. \\
\hline 1.Autonomy & - & & & & & & & & & & & & & & & & & & & \\
\hline \begin{tabular}{|l|} 
2. Craztion of a \\
Personal Nyth
\end{tabular} & $261^{* *}$ & - & & & & & & & & & & & & & & & & & & \\
\hline \begin{tabular}{|l|}
3 Easy \\
Terpperament
\end{tabular} & -029 & $.109 * 4$ & - & & & & & & & & & & & & & & & & & \\
\hline \begin{tabular}{|l|} 
4. Emotional \\
Intelligence
\end{tabular} & $207^{* *}$ & $335^{\circ * 4}$ & $.128^{* *}$ & - & & & & & & & & & & & & & & & & \\
\hline 5. Humor & .025 & $278^{* 4}$ & .018 & $348^{* 4}$ & - & & & & & & & & & & & & & & & \\
\hline \begin{tabular}{|l|} 
6. Moral \\
Development
\end{tabular} & $248^{* 4}$ & $232^{4 *}$ & $-100^{* *}$ & $327^{* 4}$ & $.125^{* *}$ & - & & & & & & & & & & & & & & \\
\hline 7. Optimism & $152^{* *}$ & $496^{* 4}$ & -.005 & $204^{* *}$ & $337^{* 4}$ & $.131^{* 4}$ & - & & & & & & & & & & & & & \\
\hline $\begin{array}{l}\text { 8. Physical } \\
\text { Besuty }\end{array}$ & .012 & $.109 * 4$ & $-124^{* 4}$ & $.174^{4 *}$ & $.448^{* *}$ & $.169 * 4$ & $.079^{*}$ & - & & & & & & & & & & & & \\
\hline 9. Self-Efficacy & $.133^{* *}$ & $345^{* *}$ & .027 & $368^{\circ *}$ & $405^{\circ *}$ & $345^{* 4}$ & $325^{\circ *}$ & $318^{* 4}$ & - & & & & & & & & & & & \\
\hline \begin{tabular}{|l|} 
10. Matemal \\
Relationship
\end{tabular} & $.179 * 4$ & $520^{\circ * 4}$ & $-.079^{*}$ & $.131^{* 4}$ & $.174^{\circ *}$ & $414^{4 *}$ & $.181^{14}$ & $.151^{* *}$ & $225^{\circ *}$ & - & & & & & & & & & & \\
\hline \begin{tabular}{|l|}
11. Patemal \\
Relationship
\end{tabular} & $263^{* *}$ & $.699 * *$ & .010 & $.156^{* *}$ & $236^{* *}$ & $467^{* * 4}$ & $.184^{* *}$ & $.091^{*}$ & $283^{* *}$ & $380^{\circ * 4}$ & - & & & & & & & & & \\
\hline \begin{tabular}{|l|}
12 Parental \\
Relationship
\end{tabular} & .057 & $237^{* 4}$ & .070 & $238^{* 4}$ & $.136^{* *}$ & $242^{* *}$ & $.199 * 4$ & $.084^{*}$ & $210^{4 *}$ & $102^{4 *}$ & $.172^{* *}$ & - & & & & & & & & \\
\hline \begin{tabular}{|l|} 
13. Parental \\
Social Support
\end{tabular} & $-100^{* 4}$ & $-266^{* 4}$ & -.063 & $-274^{* *}$ & $-136^{* *}$ & $-191^{* *}$ & $-196^{* *}$ & -.073 & $-133^{* 4}$ & $-094^{*}$ & $-171^{* *}$ & $-274^{* 4}$ & - & & & & & & & \\
\hline \begin{tabular}{|l|} 
14. History of \\
Physical Abuse
\end{tabular} & .008 & -031 & $-.168^{* 4}$ & $-124^{* *}$ & $.131^{* *}$ & .067 & -018 & $.130^{* *}$ & $.088^{\circ}$ & $.139 * 4$ & .065 & $.083^{\circ}$ & .066 & - & & & & & & \\
\hline $\begin{array}{l}\text { 15. History of } \\
\text { Sesual Abuse }\end{array}$ & .005 & .040 & -036 & .057 & $.131^{\circ *}$ & .065 & .015 & $.145^{\circ *}$ & $.094^{\circ}$ & $.115^{\circ *}$ & .059 & .065 & -.045 & $285^{\circ *}$ & - & & & & & \\
\hline \begin{tabular}{|l|} 
16. Domestic \\
Violence
\end{tabular} & -043 & -059 & -.067 & -017 & .071 & .021 & -.045 & $.101^{* *}$ & .020 & .040 & -.070 & $-.142^{* *}$ & $.092^{*}$ & $259^{* 4}$ & $355^{* 4}$ & - & & & & \\
\hline $\begin{array}{l}\text { 17. Favoritism of } \\
\text { Siblings }\end{array}$ & .007 & .016 & $-.142^{* *}$ & -032 & $.163^{* *}$ & .051 & .057 & $.137^{* 4}$ & $.099^{*}$ & $.124^{4 *}$ & .037 & -.042 & .009 & $.440^{* *}$ & $228^{\circ *}$ & $.172^{\circ *}$ & - & & & \\
\hline \begin{tabular}{|l} 
18. Parental \\
Depression
\end{tabular} & $.100^{* *}$ & .050 & $-242^{* *}$ & $.075^{*}$ & $.090^{\circ}$ & $.087^{\circ}$ & $.141^{* *}$ & $.117^{* *}$ & $.118^{* 4}$ & $.108^{* *}$ & .072 & $-089^{*}$ & $.091^{*}$ & $353^{* *}$ & $.154^{\circ *}$ & $.178^{* *}$ & $330^{\circ * 4}$ & - & & \\
\hline \begin{tabular}{|l|} 
19. Parents Not \\
Aware
\end{tabular} & $.109 * 4$ & -.047 & $-189^{* *}$ & -.043 & .068 & .062 & .021 & $.109^{* *}$ & $.077^{*}$ & $.110^{* *}$ & .059 & $-115^{* *}$ & -015 & $323^{* *}$ & $217^{* *}$ & $.154^{* *}$ & $240^{* *}$ & $3094 *$ & - & \\
\hline 20. Delinquency & -061 & -051 & -.065 & -.063 & $.198^{* *}$ & .007 & $-119^{* 4}$ & $.189 * *$ & $.096^{*}$ & .029 & .018 & -054 & .039 & $344^{* *}$ & $363^{* * 4}$ & $314^{4 *}$ & $.4900^{* 4}$ & $.493^{* 4}$ & $.439 * 4$ & - \\
\hline $\begin{array}{l}\text { 21. Intemalizing } \\
\text { Behavioo }\end{array}$ & $.107 * *$ & -059 & $-339 * 4$ & $-106^{* *}$ & .065 & .033 & $.142^{* *}$ & $.093^{*}$ & $.113^{* *}$ & $.144^{* *}$ & .040 & -064 & .017 & $555^{\circ * *}$ & $282^{* *}$ & $.175^{* *}$ & $264^{* *}$ & $193^{* *}$ & $190^{* *}$ & $246^{* *}$ \\
\hline
\end{tabular}

Figure 3. Correlations among promotive, risk and outcome variable for the full Japanese sample 
Correlations Among Promotive, Risk, and Outcome Variables for the Full Korean Sample

\begin{tabular}{|c|c|c|c|c|c|c|c|c|c|c|c|c|c|c|c|c|c|c|c|c|}
\hline & 1. & 2. & 3. & 4. & 5. & 6. & 7. & 8. & 9. & 10. & 11. & 12. & 13. & 14. & 15. & 16. & 17. & 18. & 19. & 20. \\
\hline 1.Autonomy & - & & & & & & & & & & & & & & & & & & & \\
\hline $\begin{array}{l}\text { 2. Creation of a } \\
\text { Personal Nyth }\end{array}$ & $330^{* *}$ & - & & & & & & & & & & & & & & & & & & \\
\hline \begin{tabular}{|l|} 
3Easy \\
Temperament
\end{tabular} & -.048 & $.174^{* 4}$ & - & & & & & & & & & & & & & & & & & \\
\hline 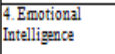 & $.111^{*}$ & $423^{* *}$ & $279 * 4$ & - & & & & & & & & & & & & & & & & \\
\hline 5. Huñor & $.146^{* *}$ & $288^{\circ *}$ & $298^{40}$ & $354^{\circ *}$ & - & & & & & & & & & & & & & & & \\
\hline \begin{tabular}{|l|} 
6. Moral \\
Development
\end{tabular} & $229^{* *}$ & $333^{\circ *}$ & $-106^{*}$ & $365^{* *}$ & $.130^{* 4}$ & - & & & & & & & & & & & & & & \\
\hline 7.0 pptimism & $247^{* *}$ & $667^{* *}$ & $216^{* 4}$ & $480^{* *}$ & $515^{* *}$ & $288^{\circ *}$ & - & & & & & & & & & & & & & \\
\hline \begin{tabular}{|l|} 
8. Physical \\
Besuty \\
\end{tabular} & $.103^{*}$ & $255^{* 4}$ & $.138^{* 4}$ & $312^{* *}$ & $421^{* *}$ & $358^{\circ * 4}$ & $.145^{* *}$ & - & & & & & & & & & & & & \\
\hline 9. Self-Efficacy & $201^{* *}$ & $484^{* *}$ & $204^{4 *}$ & $482^{* *}$ & $509^{* 4}$ & $523^{* *}$ & $369^{* *}$ & $420^{* 4}$ & - & & & & & & & & & & & \\
\hline $\begin{array}{l}\text { 10. Matemal } \\
\text { Relationship }\end{array}$ & .039 & $202^{* *}$ & .042 & $.126^{* *}$ & $302^{* *}$ & $.194^{* *}$ & .063 & .089 & $234^{* *}$ & - & & & & & & & & & & \\
\hline \begin{tabular}{|l|} 
11. Patemal \\
Relationship
\end{tabular} & .053 & .084 & .003 & $.174^{* *}$ & $252^{* *}$ & $.191^{1 *}$ & .021 & $.148^{* *}$ & $.159^{* 4}$ & $565^{\circ *}$ & - & & & & & & & & & \\
\hline $\begin{array}{l}\text { 12. Pusental } \\
\text { Relationship }\end{array}$ & .064 & $.161^{* 4}$ & .088 & $.183^{* *}$ & $278^{* 4}$ & $.2277^{44}$ & -.026 & $2077^{* 4}$ & $.145^{\circ *}$ & $595^{\circ *}$ & $597^{* 4}$ & - & & & & & & & & \\
\hline \begin{tabular}{|l|} 
13. Pasental \\
Social Support
\end{tabular} & .026 & .036 & $.117^{*}$ & .070 & $.151^{* *}$ & $.096^{*}$ & .040 & .038 & 067 & $.168^{* *}$ & $269^{4 *}$ & $261^{* *}$ & - & & & & & & & \\
\hline \begin{tabular}{|l|} 
14. History of \\
Physical Abuse
\end{tabular} & $-177^{* *}$ & $.106^{*}$ & .006 & -087 & $-149 * 4$ & .012 & -056 & -007 & -.048 & .064 & .035 & $.150^{\circ *}$ & -015 & - & & & & & & \\
\hline $\begin{array}{l}\text { 15. History of } \\
\text { Sesual Abuse }\end{array}$ & $-.121^{*}$ & .070 & .033 & $.099^{4}$ & .083 & $100^{*}$ & -031 & $-097^{*}$ & .052 & $285^{\circ *}$ & $.167^{4 * 4}$ & $.185^{\circ * 4}$ & .013 & $.174^{* 4}$ & - & & & & & \\
\hline \begin{tabular}{|l|} 
16. Domestic \\
Violence
\end{tabular} & $.103^{*}$ & .046 & .015 & $.098^{*}$ & .058 & .047 & -032 & .069 & .031 & .025 & .031 & .032 & -.057 & .025 & $.148^{* *}$ & - & & & & \\
\hline $\begin{array}{l}\text { 17. Favoritism of } \\
\text { Siblings }\end{array}$ & .025 & $.140^{* 4}$ & $.126^{* 4}$ & .077 & $.178^{* 4}$ & $.149^{* *}$ & $-.095^{*}$ & $.118^{*}$ & $.164^{* *}$ & $213^{* *}$ & $.169^{\circ * *}$ & $242^{* *}$ & $.109^{*}$ & $.099^{*}$ & $.178^{\circ *}$ & $.172^{* 4}$ & - & & & \\
\hline $\begin{array}{l}\text { 18. Pasental } \\
\text { Depression }\end{array}$ & $-115^{*}$ & .067 & $.162^{* 4}$ & $144^{* *}$ & $.116^{*}$ & .054 & .039 & .066 & $.095^{*}$ & $.156^{* *}$ & $.149 * *$ & $224^{* 4}$ & $.172^{* 4}$ & .085 & $289^{\circ * 4}$ & .074 & $208^{* 4}$ & - & & \\
\hline $\begin{array}{l}\text { 19. Parents Not } \\
\text { Aware }\end{array}$ & -.032 & $.136^{\circ 4}$ & .025 & .062 & $.119^{*}$ & .083 & -031 & .025 & $.183^{\circ}$ & $370^{* 4}$ & $314^{4 *}$ & $357^{\circ 4}$ & $.142^{* 4}$ & $.126^{4 *}$ & $346^{\circ 4}$ & .035 & $235^{\circ 4}$ & $348^{* 4}$ & - & \\
\hline 20. Delinquency & $.161^{* *}$ & $.142^{* *}$ & -.041 & $.141^{* *}$ & $229^{* 4}$ & $.174^{* *}$ & -068 & .023 & .035 & $.096^{*}$ & .030 & .083 & .074 & .076 & .087 & $352^{* *}$ & $374^{* 4}$ & $398^{\circ *}$ & $414^{* *}$ & - \\
\hline $\begin{array}{l}\text { 21. Intemalizing } \\
\text { Behavior }\end{array}$ & $-140^{* *}$ & $.1977^{\circ 4}$ & $277^{\circ 4}$ & .038 & $.115^{*}$ & $.116^{*}$ & .003 & $.133^{* 4}$ & $.181^{* *}$ & $289^{\circ *}$ & $264^{\circ *}$ & $442^{* *}$ & $.180^{* 4}$ & $214^{4 *}$ & $281^{\circ *}$ & $-118^{4}$ & $.164^{* 4}$ & .023 & .039 & -033 \\
\hline
\end{tabular}

Figure 4. Correlations among promotive, risk and outcome variable for the full Korean sample

\subsection{Multivariate Analysis}

\subsubsection{Multivariate Regression Analyses for Internalizing Behavior in the Full Sample}

A multiple regression analysis was conducted to evaluate how well the individual and family promotive and risk factors predicted internalizing behavior in the full sample. The linear combination of individual and family promotive and risk factors were significantly related to internalizing behavior and explained a significant proportion of the variance in internalizing behavior, $\mathrm{R}^{2}=.739, \mathrm{~F}(19,1712)=255.521, \mathrm{p}<.000$. From this equation it was found that easy temperament $(\beta=-.063, \mathrm{p}<.001)$, emotional intelligence $(\beta=-.050, \mathrm{p}<.041)$, moral development $(\beta=.082, \mathrm{p}<.000)$, physical beauty $(\beta=.034, \mathrm{p}<.031)$, self-efficacy $(\beta=.067$, $\mathrm{p}<.002)$, maternal relationship $(\beta=.087, \mathrm{p}<.000)$, parental relationship $(\beta=.035, \mathrm{p}<.017)$, history of physical abuse $(\beta=.161, \mathrm{p}<.000)$, history of sexual abuse $(\beta=.151, \mathrm{p}<.000)$, favoritism of siblings $(\beta=.125, \mathrm{p}<.000)$, parental depression $(\beta=.218, \mathrm{p}<.000)$ and parents not aware $(\beta=.170, p<.000)$ had a significant impact on internalizing behavior. It was found that the lower the scores on easy temperament and emotional intelligence the more likely youth will have internalizing behaviors. The higher the score on moral development, physical 
beauty, self-efficacy, maternal relationship, parental relationship, history of physical abuse, history of sexual abuse, favoritism of siblings, parental depression and parents not aware the more likely youth will have internalizing behaviors. Autonomy, creation of a personal myth, humor, optimism, paternal relationship, parental social support, and domestic violence were not predictors of depression.

\subsubsection{Multivariate Regression Analyses for Internalizing Behavior in the Full Chinese Sample}

A multiple regression analysis was conducted to evaluate how well the individual and family promotive and risk factors predicted internalizing behavior in the Chinese sample. The linear combination of individual and family promotive and risk factors were significantly related to internalizing behavior and explained a significant proportion of the variance in internalizing behavior, $\mathrm{R}^{2}=.393, \mathrm{~F}(19,634)=21.617, \mathrm{p}<.000$. From this equation it was found that easy temperament $(\beta=-.263, \mathrm{p}<.000)$, moral development $(\beta=.084, \mathrm{p}<.020)$, maternal relationship $(\beta=.073, \mathrm{p}<.015)$, parental relationship $(\beta=-.068, \mathrm{p}<.014)$, history of physical abuse $(\beta=.070, p<.0023)$, history of sexual abuse $(\beta=.108, p<.044)$, parental depression $(\beta$ $=.143, \mathrm{p}<.000)$ and parents not aware $(\beta=.117, \mathrm{p}<.000)$ had a significant impact on internalizing behavior. It was found that the lower the score on easy temperament and paternal relationship the more likely youth will have internalizing behaviors. The higher the score on moral development, maternal relationship, history of physical abuse, history of sexual abuse, parental depression and parents not aware the more likely youth will have internalizing behaviors. Autonomy, creation of a personal myth, emotional intelligence, humor, optimism, physical beauty, self-efficacy, parental relationship, paternal relationship, parental social support, domestic violence and favoritism of siblings were not predictors of internalizing behaviors.

\subsubsection{Multivariate Regression Analyses for Internalizing Behavior in the Full Japanese Sample}

A multiple regression analysis was conducted to evaluate how well the individual and family promotive and risk factors predicted internalizing behavior in the Japanese sample. The linear combination of individual and family promotive and risk factors were significantly related to internalizing behavior and explained a significant proportion of the variance in internalizing behavior, $\mathrm{R}^{2}=.570, \mathrm{~F}(19,617)=43.088, \mathrm{p}<.000$. From this equation it was found that creation of a personal myth $(\beta=-.092, \mathrm{p}<.040)$, easy temperament $(\beta=-.135, \mathrm{p}<.000)$, emotional intelligence $(\beta=-.119, \mathrm{p}<.002)$, moral development $(\beta=.087, \mathrm{p}<.007)$, self-efficacy $(\beta=.090, p<.003)$, history of physical abuse $(\beta=.216, p<.000)$, history of sexual abuse $(\beta=.118, p<.000)$, favoritism of siblings $(\beta=.125, p<.000)$, parental depression $(\beta=.235, \mathrm{p}<.000)$ and parents not aware $(\beta=.141, \mathrm{p}<.000)$ had a significant impact on internalizing behavior. It was found that the lower the score on creation of a personal myth, easy temperament and emotional intelligence the more likely youth will have internalizing behaviors. The higher the score on moral development, self-efficacy, history of physical abuse, history of sexual abuse, favoritism of siblings, parental depression and parents not aware the more likely youth will have internalizing behaviors. Autonomy, humor, optimism, physical beauty, maternal relationship, paternal relationship, parental relationship, parental 


\section{Macrothink \\ International Journal of Social Work \\ ISSN 2332-7278 \\ 2015, Vol. 2, No. 2}

social support, and domestic violence were not predictors of internalizing behaviors.

5.2.4 Multivariate Regression Analyses for Internalizing Behavior in the Full Korean Sample

A multiple regression analysis was conducted to evaluate how well the individual and family promotive and risk factors predicted internalizing behavior in the Korean sample. The linear combination of individual and family promotive and risk factors were significantly related to internalizing behavior and explained a significant proportion of the variance in internalizing behavior, $\mathrm{R}^{2}=.488, \mathrm{~F}(19,421)=21.077, \mathrm{p}<.000$. From this equation it was found that autonomy $(\beta=-.085, \mathrm{p}<.007)$, creation of a personal myth $(\beta=.126, \mathrm{p}<.018)$, easy temperament $(\beta=.146, p<.000)$, parental relationship $(\beta=.162, p<.000)$, history of physical abuse $(\beta=.068, p<.027)$, history of sexual abuse $(\beta=.182, p<.010)$, domestic violence $(\beta=$ $-.222, \mathrm{p}<.000)$, favoritism of siblings $(\beta=.140, \mathrm{p}<.000)$, parental depression $(\beta=.137$, $\mathrm{p}<.000)$ and parents not aware $(\beta=.110, \mathrm{p}<.000)$ had a significant impact on internalizing behavior. It was found that the lower the score on autonomy and domestic violence the more likely youth will have internalizing behaviors. The higher the score on creation of a personal myth, easy temperament, parental relationship, history of physical abuse, history of sexual abuse, domestic violence, favoritism of siblings, parental depression and parents not aware the more likely youth will have internalizing behaviors. Emotional intelligence, humor, optimism, moral development, physical beauty, self-efficacy, maternal relationship, paternal relationship, and parental social support were not predictors of internalizing behaviors.

Finally, a figure was created to show the best regression equation for the outcome variable by entire sample, and then for the entire sample by gender, and then for the entire sample by country, and then again by country and gender (Figure 5). 


\section{Macrothink}

\begin{tabular}{|c|c|c|c|c|}
\hline $\begin{array}{l}\text { Outcome } \\
\text { Variable }\end{array}$ & Sample & Equation & $\mathrm{R}^{2}$ & $\begin{array}{l}\mathrm{F} \text { Value and } \\
\text { Significance }\end{array}$ \\
\hline $\begin{array}{l}\text { Internalizing } \\
\text { Behavior }\end{array}$ & Full Sample & $\begin{array}{l}\text { Internalizing Behavior }=.380 \\
-.055 \text { Easy Temperament } \\
+.121 \text { Moral Development } \\
+.047 \text { Physical Beauty } \\
+.177 \text { History of Physical Abuse } \\
+.150 \text { History of Sexual Abuse } \\
+.128 \text { Favoritism of Siblings } \\
+.199 \text { Parental Depression } \\
+.178 \text { Parents not Aware }\end{array}$ & .732 & $\begin{array}{l}596.830 \\
\mathrm{p}<.000\end{array}$ \\
\hline $\begin{array}{l}\text { Internalizing } \\
\text { Behavior }\end{array}$ & $\begin{array}{l}\text { Chinese } \\
\text { Sample }\end{array}$ & $\begin{array}{l}\text { Internalizing Behavior }=2.926 \\
-.098 \text { Creation of a Personal Myth } \\
-.292 \text { Easy Temperament } \\
-.052 \text { Humor } \\
+.098 \text { Moral Development } \\
-.059 \text { Optimism } \\
+.071 \text { History of Physical Abuse } \\
+.108 \text { History of Sexual Abuse } \\
+.151 \text { Parental Depression } \\
+.131 \text { Parents not Aware }\end{array}$ & .400 & $\begin{array}{l}85.043 \\
\mathrm{p}<.000\end{array}$ \\
\hline $\begin{array}{l}\text { Internalizing } \\
\text { Behavior }\end{array}$ & $\begin{array}{l}\text { Japanese } \\
\text { Sample }\end{array}$ & $\begin{array}{l}\text { Internalizing Behavior }=.638 \\
-.142 \text { Easy Temperament } \\
-.105 \text { Emotional Intelligence } \\
+.125 \text { Moral Development } \\
+.220 \text { History Physical Abuse } \\
+.103 \text { History of Sexual Abuse } \\
+.135 \text { Favoritism of Siblings } \\
+.213 \text { Parental Depression } \\
+.144 \text { Parents not Aware }\end{array}$ & .552 & $\begin{array}{l}99.941 \\
\mathrm{p}<.000\end{array}$ \\
\hline $\begin{array}{l}\text { Internalizing } \\
\text { Behavior }\end{array}$ & $\begin{array}{l}\text { Korean } \\
\text { Sample }\end{array}$ & $\begin{array}{l}\text { Internalizing Behavior }=.824 \\
+.092 \text { Physical Beauty } \\
+.069 \text { Parental Relationship } \\
+.112 \text { History of Physical Abuse } \\
+.162 \text { Favoritism of Siblings } \\
+.196 \text { Parental Depression } \\
+.153 \text { Parents not Aware }\end{array}$ & .348 & $\begin{array}{l}38.671 \\
\mathrm{p}<.000\end{array}$ \\
\hline
\end{tabular}

Figure 5. Best fitting regression equation for each variable by full sample and gender using unstandardized coefficients

\subsection{Research Question \#1 - Individual Factors.}

What individual factors that have been shown to be important to the development of North American, European, and Australian youth impact the outcome of internalizing behavior in Chinese, Korean and Japanese youth? 


\section{MInstitute Macrothink $_{\text {Int }}$}

There seem to be many North American, European, and Australian individual factors that impact the outcomes of internalizing behavior in Chinese, Japanese, and Korean youth.

\subsubsection{Internalizing behaviors and individual factors}

Chinese youth who had not created a personal myth were more likely to have internalizing behaviors. Scoring low on easy temperament was predictive of having internalizing behaviors in Chinese and Japanese youth. For Japanese youth scoring low on emotional intelligence was predictive of have internalizing behaviors. Not having a sense of humor was predictive of internalizing behaviors in Chinese youth. It was shown in Chinese and Japanese youth that not being optimistic was predictive of internalizing behaviors. A high level of physical beauty was predictive of internalizing behaviors in Korean youth. Having a history of physical abuse was predictive of internalizing behaviors in Chinese, Japanese, and Korean youth. Finally, a high score on history of sexual abuse was predictive of Chinese and Japanese youth having internalizing behaviors.

\subsection{Research Question \#2 - Family Factors}

What family factors that have been shown to be important to the development of North American, European, and Australian youth impact the outcome of internalizing behavior in Chinese, Korean and Japanese youth?

\subsubsection{Internalizing Behavior and Family Factors}

For Japanese and Korean youth having parents favor siblings was predictive of internalizing behaviors. Scoring high on parental relationship was predictive of internalizing behaviors in Korean youth. Scoring high on parental depression was also predictive of internalizing behaviors in Chinese, Japanese, and Korean youth. Having parents who are not aware of what youth are experiencing is also predictive of internalizing behaviors for Chinese, Japanese, and Korean youth. . All of these predictors act for Asian youth as the do for American, European and Australian youth.

\subsection{Research Question \#3 - Differences by Gender for Individual Factors}

Are there differences in individual factors that impact the outcomes of internalizing behavior by gender in Chinese, Korean and Japanese youth?

\subsubsection{Internalizing Behavior, Individual Factors and Gender}

Internalizing behavior was predicted by a low score on easy temperament in Chinese and Japanese female youth. Chinese and Japanese female youth who had a high level of morality were more likely to have internalizing behaviors. A high level of physical beauty was predictive of internalizing behaviors in Chinese and Korean female youth. Finally, scoring high on history of physical abuse and history of sexual abuse was predictive of internalizing behaviors for Chinese, Japanese, and Korean female youth.

Internalizing behavior was predicted by a high score on autonomy in Chinese male youth. Chinese and Japanese male youth who had a low score on easy temperament were more likely to have internalizing behaviors. For Chinese, Japanese, and Korean male youth being 
morally developed was a predictor of internalizing behavior. Finally, scoring high on history of physical abuse and history of sexual abuse was predictive of internalizing behaviors for Chinese, Japanese, and Korean male youth.

\subsection{Research Question \#4 - Differences by Gender for Family Factors}

Are there differences in family factors that impact the outcomes of internalizing behavior by gender in Chinese, Korean and Japanese youth?

\subsubsection{Internalizing Behaviors, Family Factors and Gender}

For Chinese, Japanese, and Korean female youth having parents favor siblings was predictive of internalizing behaviors. Scoring high on parental depression was also predictive of internalizing behaviors in Chinese, Japanese, and Korean female youth. Finally, having parents who are not aware of what youth are experiencing is predictive of internalizing behaviors in Chinese, Japanese, and Korean youth. All of these predictors act as expected for Asian youth as they do for American, European and Australian youth.

For Chinese, Japanese, and Korean male youth having parents favor siblings was predictive of internalizing behaviors. Scoring high on parental depression was also predictive of internalizing behaviors in Chinese, Japanese, and Korean male youth. Finally, having parents who are not aware of what youth are experiencing is predictive of internalizing behaviors in Chinese, Japanese, and Korean male youth. All of these predictors act as expected for Asian youth as they do for American, European and Australian youth.

\section{Discussion}

This research is one of the first to investigate individual and family promotive and risk factors of Chinese, Japanese, and Korean youth that are associated with internalizing behaviors. There are many aspects of East Asian culture that are unique, however, this study found that out of the 19 variables that were examined, 15 of the variables were predictive.

\subsection{Significant Promotive Factors}

\subsubsection{Autonomy}

Being an autonomous and independent individual was found to be predictive of internalizing behaviors in the Chinese male sample. Although it has long been discussed that autonomy often appears to be a positive developmental change for adolescents for those adolescents who do not have the social skills or emotional balance to handle the change or who have families who are experiencing dysfunction (Allen et al., 2002; Allen, Hauser, Bell \& O'Conner, 1994; Steinberg, 1990) becoming more autonomous can result in negative behaviors (Allen et al., 2002; McElhaney \& Allen, 2001).

\subsubsection{Creation of a Personal Myth}

Youth who had not created a personal myth, or a plan of who they are and where they are going, was found to be a predictor of having internalizing behavior in the full Chinese sample. Acting as a guide and a plan for youth, having a personal myth was found to be an important promotive factor for resilient outcomes (Erikson, 1968; Garbarino, Dubrow, Kostelny, \& Pardo, 1992; 
Garmezy, 1996; Hartner, 1999; Howard, Dryden, \& Johnson, 1999; Kroger, 2000; Kumpfer, 1999; McAdams, 1993; McLean, 2005; Wang, Haertel, \& Walberg, 1994).

\subsubsection{Easy Temperament}

Youth who had decreased easy temperament were found to be predictive of internalizing behaviors in the full sample, Chinese sample, Japanese sample, Chinese female sample, Japanese female sample, full male sample, male Chinese sample, and male Japanese sample.

\subsubsection{Emotional Intelligence}

Internalizing behaviors were predicted by having low emotional intelligence in the full Japanese sample.

\subsubsection{Humor}

Humor was found to be a promotive factor for internalizing behaviors in the Chinese sample. Chinese youth who were funny were protected from internalizing behaviors.

\subsubsection{Moral Development}

Moral development is helpful for adolescents as it allows for youth to be able to recognize trouble and to avoid it, making choices because it is the right thing to do. It has been found in several research studies that youth who are morally developed had better outcomes (Brooks and Goldstein, 2001; Jessor, Van Den Bos, Vanderryn, Costa \& Turbin, 1995; Kumpfer, 1999). However, contrary to expectations, being highly moral was predictive of internalizing behaviors in the full sample, Chinese sample, Chinese female sample, Japanese female sample, full male sample, Chinese male sample, Japanese male sample and Korean male sample.

\subsubsection{Optimism}

Optimism, or having a lack of optimism, has a profound impact on youth's perception and there for the resilience outcomes.

\subsubsection{Physical Beauty}

In this study, increased physical beauty was a predictor of internalizing behaviors for the full sample, the Korean sample, Chinese female sample, Korean female sample.

\subsubsection{Maternal and Paternal Relationship}

Paternal relationship was not found to be a significant predictor in this study, which may be explained by the Asian work ethic of men working long hours away from home and the delineation of parenting as a more female endeavor. Though maternal relationship was found to be predictive.

\subsubsection{Parental Relationship}

Typically, parents having a good relationship has been shown to be a promotive factor for youth (Cicchetti \& Lynch, 1993; Emery \& Forehand, 1996; Rutter, 1989, 1999; Werner, 1986). However for the Korean sample and the Korean female sample that the opposite was true, parents who had a good relationship was predictive of internalizing behaviors. It is possible that youth who have parents who have a strong and dynamic relationship do not receive as much attention from their parents and feel rejected. Negative experiences, such as parental rejection, can create for youth feelings of unworthiness resulting in impaired self-esteem, depressive feelings, and a negative world view (Rohner, 2004). 


\subsection{Significant Risk Factors}

As we know these effects are harmful to North American, European, and Australian youth, however they appear to be just as detrimental to East Asian youth.

\subsubsection{History of Physical Abuse}

However, it was not predictive for the full sample of males, Chinese sample of males and the Japanese sample of males and this could possibly be explained by the concept of corporal punishment. Although, corporal punishment has often been linked to detrimental outcomes for youth (Cohen \& Brooks, 1994; McCord, 1991, 1997; Straus, 1991 1994) it has also been found to be an effective deterrent in negative youth behavior especially when it is accepted widely in the culture such as in East Asian culture (Deater-Deckard \& Dodge, 1997; Gunnoe \& Mariner, 1997). Turner \& Muller (2004) found that levels of corporal punishment differs by gender, and that males often experience greater levels of corporal punishment. This being said it is possible that males, especially East Asian males expect a certain level of physical abuse (corporal punishment), and therefore do not experience some of the harshest punishment as physical abuse.

\subsubsection{History of Sexual Abuse}

Having a history of sexual abuse was predictive of internalizing behaviors in all of the samples except the full Korean sample. Research has shown that having a history of sexual abuse has been shown to be a risk factor for people across a variety of demographic characteristics including age, gender, race and ethnicity (Beitchman, Zucker, Hood, daCosta, \& Akman, 1991; Beitchman, Zucker, Hood, daCosta, Akman, Cassavia, 1992). This phenomenon that is predictive in North American, European, and Australian youth is also a powerful predictor of internalizing behaviors in East Asian youth.

\subsubsection{Parental Depression}

Parental depression predicted internalizing behaviors. The results of this study show that not only is this true to North American, Europe and Australian youth but having a parent who is depressed is also a predictor internalizing behaviors for East Asian youth.

\subsubsection{Favoritism of Siblings}

Favoritism of siblings was predictive of internalizing behaviors. Literature regarding family treatment of siblings during childhood has demonstrated convincingly that favoritism of siblings has consequences (Suitor, et al., 2008).

\subsubsection{Parents Not Aware}

Barber, Olsen, and Shagle (1994) found that parental monitoring was associated with adolescent well-being, specifically that a lack of parental control was associated with internalizing problems. Parents not aware predicted internalizing behaviors for East Asian youth in this study. Demonstrating that parents not aware is a predictor of internalizing behaviors.

\subsection{Cultural Interpretation of the Findings}

\subsubsection{Internalizing Behavior}

The association between not having an easy temperament and moral development and internalizing behaviors was strongly significant. Youth who had a difficult temperament were 
found to have greater internalizing behaviors. Those youth who scored higher on moral development were also more likely to experience internalizing behaviors. Because of this youth with higher moral development, and youth with difficult temperaments may be at risk for internalizing behaviors. Historically, East Asian culture is one of tradition (Fisk et al., 1998; Markus \& Kityama, 1994) with an overall cultural goal of group harmony (Hsu, 1948; Yang, 1981). Youth are taught to be true to these traditions and responsive to the standards of proper behavior, particularly behaviors which benefits the group (Fisk et al., 1998; Markus \& Kitayama, 1994). East Asian youth are changing and becoming more independent (Bai, 1998; Dator \& Seo, 2004; Jin, 2003; Kinsella, 1998; Yu, 1997). As a result, youth are struggling with this shifting culture they are participating and their traditional values because it leaves them outside of the larger society.

\subsection{Limitations of the Research}

The research is based on youth self-report. The advantage is that the researcher obtains the youth's point of view, however the disadvantage is that there can be bias due to only listening to the youth's point of view.

Finally, the results of three surveys were combined, the largest group being Chinese youth. Because this sample is larger than the other samples, the data was influenced by this group's results in the overall samples.

\subsection{Direction for Future Research}

Using the survey with other Asian youth living in Thailand, Vietnam, Hong Kong, or Taiwan would give further insight into the risk and promotive factors in Asian youth. The survey has been used in the United States, Senegal, and Ghana, and further use of the survey would enhance our knowledge of promotive and risk factors for internalizing behavior that are universal or more influenced by culture, region or gender.

\section{References}

Allen, J. P., Marsh, P., McFarland, C., McElhaney, K. B., Land, D. J., Jodl, K. M., \& Peck, S., (2002). Attachment and autonomy as predictors of the development of social skills and delinquency during midadolescence. Journal of Counseling and Clinical Psychology, 70(1), 56-66. http://dx.doi.org/10.1037/0022-006X.70.1.56

Ammerman, R. T., Cassisi, J. E., Hersen, M., \& Van Hasselt, V. B. (1986). Consequences of physical abuse and neglect in children. Clinical Psychology Review, 6, 291-310. http://dx.doi.org/10.1016/0272-7358(86)90003-6

Amoato, P. A., \& Gilbreth, J. G. (1999). Nonresident fathers and children's well-being: Meta analysis. Journal of Marriage and the Family, 61, 15-73. http://dx.doi.org/10.2307/353560

Asarnow, J. R., Porta, G., Spirito, A., Emslie, G., Clarke, G., Wagner, K. D., ... Keller, M. (2011). Suicide attempts and nonsuicidal self-injury in the treatment of resistant depression in adolescents: findings from the TORDIA study. Journal of the American Academy of Child \& Adolescent Psychiatry, 50(8), 772-781. http://dx.doi.org/10.1016/j.jaac.2011.04.003

Austin, E. J., Saklofske, D. H., \& Egan, V. (2005). Personality, well-being, and health correlates of trait emotional intelligence. Personality and Individual Differences, 38, 547-558. http://dx.doi.org/10.1016/j.paid.2004.05.009 
Bai L. (1998). Monetary reward versus the national ideological agenda: Career choice among Chinese university students. Journal of Moral Education, 27(4), 525-541. http://dx.doi.org/10.1080/0305724980270406

Balog, S. A. (1995). Personality characteristics in children witnessing domestic violence: Externalizing behavior, internalizing behavior, social competence, and self-concept. Unpublished dissertation, University of Denver, Colorado.

Bandura, A. (1977). Self-efficacy: Toward a unifying theory of behavioral change. Psychological Review, 34(2), 191-215. http://dx.doi.org/10.1037/0033-295X.84.2.191

Beitchman, J. H., Zucker, K. J., Hood, J. E., DaCosta, G. A., \& Akman, D. (1991). A review of the short-term effects of child sexual abuse. Child abuse \& neglect, 15(4), 537-556. http://dx.doi.org/10.1016/0145-2134(91)90038-F

Beitchman, J. H., Zucker, K. J., Hood, J. E., daCosta, G. A., Akman, D., \& Cassavia, E. (1992). A review of the long-term effects of child sexual abuse. Child Abuse and Neglect, 16, 101-118. Bricker, V. R. (1980). The function of humor in Zincantan. Journal of Anthropological Research, 36(4), 411-418. http://dx.doi.org/10.1016/0145-2134(92)90011-F

Brooks, B. (1985). Sexually abused children and adolescent identity development. American Journal of Psychotherapy, 39, 401-410.

Brooks, R., \& Goldstein, S. (2001). Raising resilient children: Fostering strength, hope, and optimism in your child. Contemporary Books: Lincolnwood, IL.

Buka, S. L., Stichick, T. L., Birdthistle, I., \& Earls, F. J. (2001). Youth exposure to violence: Prevalence, risks, and consequences. American Journal of Orthopsychiatry, 71(3), 298-310. http://dx.doi.org/10.1037/0002-9432.71.3.298

Burgess, A. W., Hartman, C. R., McCausland, M. P., \& Powers, P. (1984). Response patterns in children and adolescents exploited through sex rings and pornography. American Journal of Psychiatry, 141, 656-662. http://dx.doi.org/10.1176/ajp.141.5.656

Carlton, B. S., Goebert, D. A., Miyamoto, R. H., Andrade, N. N., Hishinuma, E. S., Makini, G. K., ... Nishimura, S. T. (2006). Resilience, family adversity and well-being among Hawaiian and non-Hawaiian adolescents. International Journal of Social Psychiatry, 52(4), 291-308. http://dx.doi.org/10.1177/0020764006065136

Chaffin, M., Kelleher, K., \& Hollenberg, K. (1996). Onset of physical abuse and neglect: Psychiatric, substance abuse, and social risk factors from prospective community data. Child Abuse and Neglect, 20, 191-203. http://dx.doi.org/10.1016/S0145-2134(95)00144-1

Cicchetti, D., \& Lynch, M. (1993). Toward an ecological/transactional model of community violence and child maltreatment: Consequences for children's development. Psychiatry, 56, 96-118.

Civikly, J. M. (1986). Humor and the enjoyment of college teaching. New directions for teaching and learning, 26, 61-70. http://dx.doi.org/10.1002/tl.37219862609

Conaway, L. P., \& Hansen, D. J. (1989). Social behavior of physically abused and neglected children: A critical review. Clinical Psychology Review, 9, 627-652. http://dx.doi.org/10.1016/0272-7358(89)90015-9 


\section{Macrothink}

International Journal of Social Work ISSN 2332-7278 2015, Vol. 2, No. 2

Cronbach, L. J. (1951). Coefficient alpha and the internal structure of tests. Psychometrika, 16, 297-334. http://dx.doi.org/10.1007/BF02310555

Cummings, E. M. \& Davies, P. (1994). Children and Marital Conflict. New York: Guilford Press.

Dator, J., \& Seo, Y. (2004). Korea as the wave of a future: The emerging dream society of icons and aesthetic experience. Journal of Future Studies, 9(1), 31-44.

Deater-Deckard, K. (2000). Parenting and child behavioral adjustment in early childhood: A quantitative genetic approach to studying family processes. Child Development, 71, 468-484. http://dx.doi.org/10.1111/1467-8624.00158

Deater-Deckard, K., \& Kenneth, A. D. (1997). Externalizing behavior problems and discipline revisited: Nonlinear effects and variation by culture, context, and gender. Psychological Inquiry, 8, 161-175. http://dx.doi.org/10.1207/s15327965pli0803_1

Dixon, L., Hamilton-Giachritsis, C., \& Browne, K. (2005a). Attributions and behaviors of parents abused as children: A mediational analysis of the intergenerational continuity of child maltreatment (Part II). Journal of Child Psychology and Psychiatry, 46(1), 58-68. http://dx.doi.org/10.1111/j.1469-7610.2004.00340.x

Dixon, L., Hamilton-Giachritsis, C., \& Browne, K. (2005b). Risk factors of parents abused as children: A meditational analysis of the intergenerational continuity of child maltreatment (Part I). Journal of Child Psychology and Psychiatry, 46(1), 47-57. http://dx.doi.org/10.1111/j.1469-7610.2004.00340.x

Dodge, K. A., \& Garber, J. (1991). Emotion and social information processing. In J. Garber \& K. A. Dodge (Eds.), The development of emotion regulation and dysregulation (pp. 159-181). Cambridge, England: Cambridge University Press. http://dx.doi.org/10.1017/CBO9780511663963.009

Dunn, J., Stocker, C., \& Plomin, R. (1990). Nonshared experiences within the family: Correlates of behavioral problems in middle childhood. Development and Psychopathology, 2, 113-126. http://dx.doi.org/10.1017/S0954579400000651

Dweck, C. S., \& Licht, B. G. (1980). Learned helplessness and intellectual achievement. In J. Graber \& M. E. P. Seligman (Eds.), Human helplessness: Theory and application (pp. 197-221). New York: Academic Press.

Edleson, J. (1999). Children's witnessing of adult domestic violence. Journal of Interpersonal Violence, 14(8), 839-870. http://dx.doi.org/10.1177/088626099014008004

Emery, R. E. (1988). Marriage, divorce, and children's adjustment. Newbury Park, CA: Sage. Emery, R. E., \& Forehand, R. (1996). Parental divorce and children's well-being: A focus on resilience. In R.J. Haggerty, L.R. Sherrod, N. Garmezy, \& M. Rutter (Eds.), Stress, risk and resilience in children and adolescents: Processes, mechanisms, and interventions (pp. 64-99). New York: Cambridge University Press.

Erikson, E. (1968). Identity: Youth and crisis. New York: Norton.

Famularo, R., Kinscherff, R., \& Fenton, T. (1992). Psychiatric disgnoses of abusive mothers: A preliminary report. The Journal of Nervous and Mental Disease, 180(10), 658-661. http://dx.doi.org/10.1097/00005053-199210000-00008 
Fantuzzo, J. W. (1990). Behavioral treatment of the victims of child abuse and neglect. Behavioral Modification, 14, 316-339. http://dx.doi.org/10.1177/01454455900143006

Fantuzzo, .J W., \& Lindquist, C. U. (1989). The effects of observing conjugal violence on children: A review and analysis of research methodology. Journal of Family Violence, 4(1), 77-94. http://dx.doi.org/10.1007/BF00985658

Fantuzzo, J., \& Mohr, W. K. (1999). Prevalence and effects of child exposure to domestic violence. The Future of Children, 9(3), 21-32. http://dx.doi.org/10.2307/1602779

Faust, M. S. (1983). Alternative constructions of adolescent growth. In J. Brooks-Gunn \& A. C. Petersen (Eds.), Girls at puberty: Biological and psychological perspectives (pp. 105-125). New York: Plenum Press. http://dx.doi.org/10.1007/978-1-4899-0354-9_6

Feldman, S. S., \& Rosenthal, D. A. (1991). Age expectation of behavioral autonomy in Hong Kong, Australian and American youth: The influence of family variables and adolescents' values. International Journal of Psychology, 26(1), 1-23. http://dx.doi.org/10.1080/00207599108246846

Feinberg, M., \& Hetherington, E. M. (2001). Differential parenting as a within-family variable. Journal of Family Psychology, 22-37. http://dx.doi.org/10.1037/0893-3200.15.1.22

Feiring, C., \& Taska, L. (2005). The persistence of shame following sexual abuse: A longitudinal look at risk and recovery. Child Maltreatment, 10(4), 337-349. http://dx.doi.org/10.1177/1077559505276686

Fergusson, D. M., Horwood, L., \& Lynskey, M. T. (1996). Childhood sexual abuse and psychiatric disorder in young adulthood: II. Psychiatric outcomes of childhood sexual abuse. Journal of the American Academy of Child \& Adolescent Psychiatry, 35(10), 1365-1374. http://dx.doi.org/10.1097/00004583-199610000-00024

Field, T. (1984). Early interactions between infants and their postpartum depressed mothers. Infant Behavior and Development, 7, 527-532. http://dx.doi.org/10.1016/S0163-6383(84)80179-4

Fincham, F. D., Hokoda, A., \& Sanders, R. (1989). Learned helplessness, test anxiety, and academic achievement: A longitudinal analysis. Child Development, 60(1), 138-145. http://dx.doi.org/10.2307/1131079

Fisher, S. (1990). The evolution of psychological concepts about the body. In T. F. Cash \& T. Pruzinsky (Eds.), Body images (pp. 3-20). New York: The Guilford Press.

Fisk, W. J., Faulkner, D., Sullivan, M., Dong, C., Dabrowski, J. M., Thomas Jr., M. J., ... Boeinger, M. (1998). The healthy building intervention study: Objectives, methods, and results of selected environmental measurements, LBNJ-41546, Lawrence Berkeley National Laboratory, Berkeley, CA.

Flook, L., Repetti, R. L., \& Ullman, J. B. (2005). Classroom social experiences as predictors of academic performance. Developmental Psychology, 41, 319-327. http://dx.doi.org/10.1037/0012-1649.41.2.319

Garbarino, J., Dubrow, N., Kostelny, K., \& Pardo, C. (1992). Children in danger: Coping with the effects of community violence. San Francisco: Jossey-Bass. 
Garmezy, N. (1996). Reflections and commentary on risk, resilience and development. In R. Haggerty, L. Sherrod, N. Garmezy \& M. Rutter (Eds.) Stress, risk, and resilience in children and adolescents (pp. 1-18). Cambridge, UK: Cambridge University Press.

Gillham, J., \& Reivich, K. (2004). Cultivating optimism in childhood and adolescence. The Annals of the American Academy of Political and Social Science, 591(1), 146-163. http://dx.doi.org/10.1177/0002716203260095

Goleman, D. (1995). Emotional intelligence: Why it can matter more than IQ. NewYork: Bantam Books.

Goleman, D. (1998). An EI-based theory of performance. In C. Cherniss \& D. Goleman (Eds.) The emotionally intelligent workplace: How to select for, measure, and improve emotional intelligence in individuals, groups, and organizations (pp. 27-44). San Francisco, CA: Jossey-Bass.

Goleman, D. (2001). The emotionally intelligent workplace. San Francisco: Jossey-Bass.

Golombok, S. (2000). Parenting: What really counts? New York: Routledge.

Gomes-Schwartz, B., Horowitz, J. M., \& Sauzier, M. (1985). Severity of emotional distress among sexually abused preschool, school-age, and adolescent children. Hospital and Community Psychiatry, 36(5), 503-508. http://dx.doi.org/10.1176/ps.36.5.503

Gore, S., \& Eckenrode, J. (1996). Context and process in research on risk and resilience. In R. Haggerty, L. Sherrod, N., Garmezy, \& M. Rutter (Eds.), Stress, risk, and resilience in children and adolescents (pp. 19-63). Cambridge University Press.

Gottman, J. M. (1979). Marital interaction: Experimental investigations. San Diego, CA: Academic Press.

Gottman, J. M. (1994). What predicts divorce? Hillsdale, NJ: Lawernce Erlbaum Associates.

Graham-Bermann, S. A., \& Seng, J. (2005). Violence exposure and traumatic stress symptoms as additional predictors of health problems in high-risk children. The Journal of pediatrics, 146(3), 349-354. http://dx.doi.org/10.1016/j.jpeds.2004.10.065

Gresham, F. M., \& Kern, L. (2004). Internalizing behavior problems in children and adolescents. In R. B. Rutherford, M. M. Quinn, \& S. R. Mathur (Eds.) Handbook of research in emotional and behavioral disorders (pp. 262-281). New York, NY: Guilford Press.

Gunnoe, M. L. \& Mariner, C. L. (1998). Toward a developmental-contextual model of the effects of parental spanking on children's aggression. Archives of Pediatric and Adolescent Medicine, 151, 768-775. http://dx.doi.org/10.1001/archpedi.1997.02170450018003

Handlin, O. H. \& Handlin, M. F. (1971). Facing life: Youth and the family in American history. Boston: Little-Brown.

Hankin, B. L. (2006). Adolescent depression: description, causes, and interventions. Epilepsy \& Behavior, 8(1), 102-114. http://dx.doi.org/10.1016/j.yebeh.2005.10.012

Harris, I., \& Howard, K. (1984). Parental criticism and the adolescent experience. Journal of Youth and Adolescence, 13, 113-121. http://dx.doi.org/10.1007/BF02089105

Harter, S. (1990). Developmental differences in the nature of self-representations: Implications for the understanding assessment and treatment of maladaptive behavior. Cognitive Therapy and Research, 14, 113-142. http://dx.doi.org/10.1007/BF01176205 
Heller, T. L., \& Baker, B. L. (2000). Maternal negativity in children's externalizing behavior. Early Education and development, 11, 483-498. http://dx.doi.org/10.1207/s15566935eed1104_7

Hetherington, E. (1989). Coping with family transitions. Child Development, 60, 1-14. http://dx.doi.org/10.2307/1131066

Howard, S., Dryden, J., \& Johnson, B. (1999). Childhood resilience: Review and critique of the literature. Oxford Review of Education, 25(3), 307-324. http://dx.doi.org/10.1080/030549899104008

Hsu, F. K. L. (1948). Under the ancestor's shadow: Chinese culture and personality. New York: Columbia University Press.

Izard, C., Fine, S., Schultz, D., Mostow, A., Ackerman, B., \& Youngstrom, E. (2001). Emotion knowledge as a predictor of social behavior and academic competence in children at risk. Psychological Science, 12(1), 18-23. http://dx.doi.org/10.1111/1467-9280.00304

Jacob, T., \& Johnson, S. L. (1997). Parent child interaction among depressed fathers and mothers: Impact on child functioning. Journal of Family Psychology, 11, 391-409. http://dx.doi.org/10.1037/0893-3200.11.4.391

Jessor, R., Van Den Bos, J., Vanderryn, J., Costa, F. M., \& Turbin, M. S. (1995). Protective factors in adolescent problem behavior: Moderator effects and developmental change. Developmental Psychology, 31(6), 923-933. http://dx.doi.org/10.1037/0012-1649.31.6.923

Jin, Q. (2003). The secularization of modern Chinese youth, Chinese Education and Society, 35(6), 24-32. http://dx.doi.org/10.2753/CED1061-1932350624

Kandel, E., Mednick, S. A., Kirkegaard-Sorenson, L., Hutchings, E., Knop, J., Rosenberg, R., \& Schusinger, F. (1988). IQ as a protective factor for subjects at high risk for antisocial behavior. Journal of Consulting and Clinical Psychology, 56, 224-226. http://dx.doi.org/10.1037/0022-006X.56.2.224

Kim-Cohen, J. Moffit, T. E., Taylor, A., Pawlby, S. J., \& Caspi, A. (2005). Maternal depression and children's antisocial behavior: Native and nurture effects. Archives of General Psychiatry, 62, 173-181. http://dx.doi.org/10.1001/archpsyc.62.2.173

Kinsella, S. (1998). Japanese subculture in the 1990s: Otaku and the amateur manga movement. Journal of Japanese Studies, 24(2), 289-316. http://dx.doi.org/10.2307/133236

Kotch, J. B., Browne, D. C., Dufort, V., Winsor, J., \& Catellier, D. (1999). Predicting child maltreatment in the first 4 years of life from characteristics assessed in the neonatal period. Child Abuse and Neglect, 23, 305-319. http://dx.doi.org/10.1016/S0145-2134(99)00003-4

Kowal, A. K., \& Kramer, L. (1997). Children's understanding of parental differential treatment. Child Development, 68, 113-126. http://dx.doi.org/10.2307/1131929

Kroger, J. (2000). Identity development: Adolescence through adulthood. Thousand Oaks, CA: Sage.

Kumpfer, K. (1999). Factors and processes contributing to resilience: The resilience framework. In M. Glantz \& J. Johnson (Eds.), Resilience and development: Positive life adaptions (pp. 179-224). New York: Kluwer. 
Lamphear, V. S. (1985). The impact of maltreatment on children's psychosocial adjustment: A review of the research. Child Abuse and Neglect, 9, 251-263. http://dx.doi.org/10.1016/0145-2134(85)90018-3

Landy, D., \& Mettee, D. (1969). Evaluation of an aggressor as a function of exposure to cartoon humor. Journal of Personality and Social Psychology, 12(1), 66-71. http://dx.doi.org/10.1037/h0027360

Laser, J. A., Luster, T., \& Oshio, T. (2007a). Promotive and risk factors to deviant behavior in Japanese youth. Criminal Justice and Behavior, 34(11), 1463-1480. http://dx.doi.org/10.1177/0093854807306127

Laser, J. A., Luster, T., \& Oshio, T. (2007b). Risk and promotive factors related to depressive symptoms among Japanese youth. American Journal of Ortho Psychiatry, 77(4), 523-533. http://dx.doi.org/10.1037/0002-9432.77.4.523

Laser, J. A., \& Nicotera, N. (2011). Working with adolescents: A guide for practitioners. New York: Guilford Press.

Leschied, A., Chiodo D., Whitehead, P., \& Hurley, D. (2005). The relationship between maternal depression and child outcomes in a child policy welfare sample: implications for treatment and policy. Child and Family Social Work, 10, 281-291. http://dx.doi.org/10.1111/j.1365-2206.2005.00365.x

Lindberg, F. H., \& Distad, L. J. (1985). Post-traumatic stress disorders in women who experienced childhood incest. Child Abuse \& Neglect, 9, 329-334. http://dx.doi.org/10.1016/0145-2134(85)90028-6

Loeber, R., \& Stouthamer-Loeber, M. (1986). Family factors as correlates and predictors of juvenile conduct problems and delinquency. In M. Tonry \& Morris (Eds.), Crime and justice (Vol. 7, pp. 29-150). Chicago: University of Chicago Press. http://dx.doi.org/10.1086/449112 Luthar, S. S., Cicchetti, D., \& Becker, B. (2000). Research on resilience: Response to commentaries. Child Development, 71, 573-575. http://dx.doi.org/10.1111/1467-8624.00168

Malinosky-Rummell, R., \& Hansen, D. J. (1993). Long-term consequences of childhood $\begin{array}{lllll}\text { physical abuse. } \quad \text { Psychological } & \text { Bulletin, } & \text { 114(1), }\end{array}$ http://dx.doi.org/10.1037/0033-2909.114.1.68

Margolin, G., \& Gordis, E. B. (2000). The effects of family and community violence on children. Annual Review of Psychology, 51, 445-479. http://dx.doi.org/10.1146/annurev.psych.51.1.445

Markus, H. R., \& Kitayama, S. (1994). The cultural construction of self and emotion: Implications for social behavior. In S. Kitayama \& H. R. Markus (Eds.), Emotion and culture: Empirical studies of mutual influence (pp. 89-132). Washington, DC: American Psychological Association. http://dx.doi.org/10.1037/10152-003

Martin, R. A. (2001). Humor, laughter, and physical health: Methodological issues and research findings. Psychological bulletin, 127(4), 504-519. http://dx.doi.org/10.1037/0033-2909.127.4.504

Martinek, T., \& Griffith, J. B. (1993). Working with the learned helpless child. Journal of Physical Education, Recreation, and Dance, 64(6), 19-20. 
http://dx.doi.org/10.1080/07303084.1993.10609995

Masten, A. A., \& Powell, J. L. (2003). A resilience framework for research, policy, and practice. In S. S. Puthar (Ed.), Resilience and vulnerability: Adaptation I the context of childhood adversities (pp. 1-25). New York: Cambridge University Press. http://dx.doi.org/10.1017/CBO9780511615788.003

Masten, A. S. (2013). Resilience in development: Implications of the study of successful adaptation for developmental. In The emergence of a discipline: Rochester symposium on developmental psychopathology (pp. 261-292). Psychology Press. http://dx.doi.org/10.1093/oxfordhb/9780199958474.013.0023

Masten, A. S., \& Coatsworth, J. D. (1998). The development of competence in favorable and unfavorable environments: Lessons from research on successful children. American Psychologist, 53(2), 205. http://dx.doi.org/10.1037/0003-066X.53.2.205

Mavroveli, S., Petrides, K. V., Rieffe, C., \& Bakker, F. (2007). Trait emotional intelligence, psychological well-being and peer-rated social competence in adolescence. British Journal of Developmental Psychology, 25, 263-275. http://dx.doi.org/10.1348/026151006X118577

McAdams, D. P. (1993). The stories we live by: Personal myths and the making of self. New York: Morrow.

McAdams, D. P. (2001). The psychology of life stories. Review of General Psychology, 5, 100-122. http://dx.doi.org/10.1037/1089-2680.5.2.100

McElhaney, K. B., \& Allen, J. P. (2001). Autonomy and adolescent social functioning: The moderating effect of risk. Child Development, 72, 220-231. http://dx.doi.org/10.1111/1467-8624.00275

McHale, S. M., \& Gamble, W. C. (1989). Sibling relationships of children with disabled and nondisabled brothers and sisters. Developmental Psvchologv, 25, 421-429. http://dx.doi.org/10.1037/0012-1649.25.3.421

McHale, S. M., \& Pawletko, T. M. (1992). Differential treatment of siblings in two family contexts. Child Development, 63, 68-81. http://dx.doi.org/10.2307/1130902

McLean, K. C. (2005). Late adolescent identity development: Narrative meaning-making and memory telling. Developmental Psychology, 41, 683-691. http://dx.doi.org/10.1037/0012-1649.41.4.683

Merrell, K.W., \& Walker, H.M. (2004). Deconstructing a definition: Social maladjustment versus emotional disturbance and moving the EBD field forward. Psychology in the Schools, 41, 899-910. http://dx.doi.org/10.1002/pits.20046

Mullick, M., Miller, L.J.., \& Jacobson, T. (2001). Insight into mental illness and child maltreatment risk among mothers with major psychiatric disorders. Psychiatric Services, 52(4), 488-492. http://dx.doi.org/10.1176/appi.ps.52.4.488

Nezu, A. M., Nezu, C. M., \& Blissett, S. E. (1988). Sense of humor as a moderator of the relation between stressful events and psychological: A prospective analysis. Journal of $\begin{array}{llll}\text { Personality } \quad \text { and Social 5ychology, } & 54,\end{array}$ http://dx.doi.org/10.1037/0022-3514.54.3.520

Overholser, J. C. (1992). Interpersonal dependency and social loss. Personality and 
Individual Differences, 13, 17-23. http://dx.doi.org/10.1016/0191-8869(92)90212-8

Parke, R. D., Dennis, J., Flyr, M. L., Morris, K. L., Leidy, M. S., \& Schofield, T. J. (2005). Fathers: Cultural and ecological perspectives. In T. Luster \& L. Okagaki (Eds.), Parenting: An Ecological Perspective (pp. 103-146). Mahwah: NJ: Lawrence Erlbaum Associates.

Parker, J., \& Herrera, C. (1996). Interpersonal processes in friendships: A comparison of maltreated and non-maltreated children's experiences. Developmental Psychology, 32, 1025-1038. http://dx.doi.org/10.1037/0012-1649.32.6.1025

Peterson, C. (2000). The future of optimism. American Psychologist, 55(1), 44-55. http://dx.doi.org/10.1037/0003-066X.55.1.44

Peterson, G. W. (1986). Parent-youth power dimensions and the behavioral autonomy of adolescents. Journal of Adolescent Research, 1(2), 231-249. http://dx.doi.org/10.1177/074355488612008

Piko, B. F. (1998). Social support and health in adolescence: A factor analytical study. British $\begin{array}{lllll}\text { Journal of Health 333-344. } & \text { Psychology, 3, }\end{array}$ http://dx.doi.org/10.1111/j.2044-8287.1998.tb00578.x

Piko, B. F., Kovacs, E., \& Fitzpatrick, K. M. (2009). What makes a difference? Understanding the role of protective factors in Hungarian adolescents' depressive symptomatology. European child \& Adolescent Psychiatry, 18(10), 617-624. http://dx.doi.org/10.1007/s00787-009-0022-y

Pollard, J. A., Hawkins, D., \& Arthur, M. W. (1999). Risk and protection: Are both necessary to understand diverse behavioral outcomes in adolescence? Social Work Research, 23, 145-158. http://dx.doi.org/10.1093/swr/23.3.145

Rae-Grant, N., Thomas, H., Offord, D. R., \& Boyle, M. H. (1989). Risk, protective factors, and prevalence of behavioral and emotional disorders in children and adolescents. Journal of the American Academy of Child and Adolescent Psychiatry, 28, 262-268. http://dx.doi.org/10.1097/00004583-198903000-00019

Rohner, R. P. (2004). The parental "acceptance-rejection syndrome": Universal correlates of $\begin{array}{llll}\text { perceived } \quad \text { rejection. American } & \text { Psychologist, } & \text { 59, } & \text { 827-840. }\end{array}$ http://dx.doi.org/10.1037/0003-066X.59.8.830

Roth, J. L., \& Brooks-Gunn, J. (2000). What do adolescents need for healthy development? Implications for youth policy. Society for Research in Child Development Social Policy Report, 14(1), 3-19.

Rosenblum, G. D., \& Lewis, M. (1999). The relations among body image, physical attractiveness, and body mass in adolescents. Child Development, 70 (1), 50-64. http://dx.doi.org/10.1111/1467-8624.00005

Rossman, B. B. (1998). Descartes's error and posttraumatic stress disorder: Cognition and emotion in children who are exposed to parental violence. In G. W. Holden, R. Geffner, \& E. N. Jouriles (Eds.), Children exposed to marital violence (pp. 223-256), Washington, DC: American Psychological Association. http://dx.doi.org/10.1037/10257-007

Runtz, M., \& Briere, J. (1986). Adolescent "acting-out" and childhood history of sexual abuse. Journal of Interpersonal Violence, I, 326-334. 
http://dx.doi.org/10.1177/088626086001003005

Ruschena, E., Prior, M., Sanson, A. \& Smart, D. (2005). A longitudinal study of adolescent adjustment following family transitions. Journal of Child Psychology and Psychiatry, 46, 353-363. http://dx.doi.org/10.1111/j.1469-7610.2004.00369.x

Rutter, M. (1979). Protective factors in children's responses to stress and disadvantage, In M. W. Kent \& J. E. Rolf (Eds.), Primary prevention of psychopathology. III. Social competence in children (pp. 49-74). Hanover, NH: University Press of New England.

Rutter, M. (1985). Resilience in the face of adversity: Protective factors and resistance to psychiatric disorder. British Journal of Psychiatry, 147, 598-611. http://dx.doi.org/10.1192/bjp.147.6.598

Rutter, M. (1989). Pathways from childhood to adult life. Journal of Child Psychology and Psychiatry, 30(1), 23-51. http://dx.doi.org/10.1111/j.1469-7610.1989.tb00768.x

Rutter, M. (1990). Psychosocial resilience and protective mechanisms. In J. Rolf, A. Masten, D. Cicchetti, K. H. Nuechterlein, \& S. Weintraub (Eds.), Risk and protective factors in the development of psychopathology (pp. 181-214). Cambridge, England: Cambridge University Press. http://dx.doi.org/10.1017/CBO9780511752872.013

Rutter, M. (1999). Resilience concepts and findings: Implications for family therapy. Journal of Family Therapy, 21(2), 119-144. http://dx.doi.org/10.1111/1467-6427.00108

Rutter, M., Giller, H., \& Hagell, A. (1998). Antisocial behavior by young people. Cambridge, England: Cambridge University Press.

Salmon, C. A., Shackelford, T. K., \& Michalski, R. L. (2012). Birth order, sex of child, and perceptions of parental favoritism. Personality and Individual Differences, 52(3), 357-362. http://dx.doi.org/10.1016/j.paid.2011.10.033

Sameroff, A. (2000). Ecological perspectives on developmental risk. In J. Osofsky \& H. Fitgerald (Eds.), WAIMH handbook of infant mental health (Vol. 4, pp. 1-33). New York: Wiley.

Sameroff, A. J., Bartko, W. T., Baldwin, A., Baldwin, C., \& Seifer, R. (1998). Family and social influences on the development of child competence. In M. Wei \& C. Fairing (Eds.), Families, risk, and competence. Mahwah, NJ: Erlbaum.

Scholte, R. H. J., Engels, R. C. M. E., de Kemp, R. A. T., Harakeh, Z., \& Overbeek, G. (2007). Differential parental treatment, sibling relationships and delinquency in adolescence. Journal of Youth and Adolescence, 36, 661-671. http://dx.doi.org/10.1007/s10964-006-9155-1

Seligman, M. E. P. (2006a). The optimistic child. New York: Houghton Mifflin.

Seligman, M. E. P. (2006b). Learned optimism: How to change your mind and your life. New York: Vintage Books.

Shamseddeen, W., Asarnow, J. R., Clarke, G., Vitiello, B., Wagner, K. D., Birmaher, B., ... Brent, D. A. (2011). Impact of physical and sexual abuse on treatment response in the Treatment of Resistant Depression in Adolescent Study (TORDIA). Journal of the American Academy of Child \& Adolescent Psychiatry, 50(3), 293-301. http://dx.doi.org/10.1016/j.jaac.2010.11.019

Shanahan, L., McHale, S. M., Crouter, A. C., \& Osgood, D. W. (2008). Linkages between 
parents' differential treatment, youth depressive symptoms, and sibling relationships. Journal of Marriage and Family, 70, 480-494. http://dx.doi.org/10.1111/j.1741-3737.2008.00495.x

Sheppard, M., (1997). Double jeopardy: The link between child abuse and maternal depression in child and family social work. Child and Family Social Work, 2, 91-107. http://dx.doi.org/10.1046/j.1365-2206.1997.00047.x

Silvern, L., Karyl, J., Waedale, L., Hodges, W. F., Starek, J., Heidt, E., \& Min, K. (1995). Retrospective reports of parental partner abuse: Relationship to depression, trauma symptoms and self-esteem among college students. Journal of Family Violence, 10, 177-202. http://dx.doi.org/10.1007/BF02110599

Smith, C. M., \& Powell, L. (1988). The use of disparaging humor by group leaders. Southern $\begin{array}{llll}\text { Speech Communication } \quad \text { Journal, } & \text { 279-292. }\end{array}$ http://dx.doi.org/10.1080/10417948809372729

Smith, J. \& Prior, M. (1995). Temperament and stress resilience in school-age children: A within-families study. Journal of the American Academy of Child and Adolescent Psychiatry, 34, 168-179. http://dx.doi.org/10.1097/00004583-199502000-00012

Sorell, G. T. \& Nowark, C. A. (1981). The role of physical attractiveness as a contributor to individual development. In R. M. Lerner \& N. A. Busch-Rossnagel (Eds.), Individuals as producers of their development (pp. 389-446). New York: Academic Press. http://dx.doi.org/10.1016/B978-0-12-444550-5.50021-3

Stanton, B. F., Xiaoming, L., Galabraith, J., Cornick, G., Feigelman, S., Kaljee, L., \& Zhou, Y. (2000). Parental underestimates of adolescent risk behavior: A randomized, controlled trial of a parental monitoring intervention. Journal of Adolescent Health, 26, 18-26. http://dx.doi.org/10.1016/S1054-139X(99)00022-1

Straus, M. A. (1991). Discipline and deviance: Physical punishment of children and violence and other crimes in adulthood. Social Problems, 38, 133-154. http://dx.doi.org/10.2307/800524

Straus, M. A. (1994). Beating the devil out of them: Corporal punishment in American families and its effect on children. Boston: Lexington.

Steinberg, L. D. (1990). Interdependence in the family: Autonomy, conflict and harmony in the parent-adolescent relationship. In S. S. Feldman \& G. R. Elliot (Eds.), At the threshold: The developing adolescent. Cambridge, MA: Harvard University Press.

Stevenson, H. W., \& Stigler, J. W. (1992). The learning gap: Why our schools are failing and what we can learn from Japanese and Chinese education. New York: Summit Books.

Stipek, D. J., \& Kowalski, P. S. (1989). Learned helplessness in task-orienting versus performance-orienting testing conditions. Journal of Educational Psychology, 81(1), 384-391. http://dx.doi.org/10.1037/0022-0663.81.3.384

Suitor, J., Sechrist, J., Plikuhn, M., Pardo, S. T., \& Pillemer, K. (2008). Within-family differences in parent-child relations across the life course. Current Directions in Psychological Science, 17, 334-338. http://dx.doi.org/10.1111/j.1467-8721.2008.00601.x

Tschann, J., Kaiser, P., Chesner, M. A., Alkon, A., \& Boyce, W. T. (1996). Resilience and vulnerability among preschool children: Family functioning, temperament, and behavior 
problems. Journal of the American Academy of Child and Adolescent Psychiatry, 35, 184-192. http://dx.doi.org/10.1097/00004583-199602000-00012

Turner, H. A., \& Muller, P. A. (2004). Long-term effects of child corporal punishment on depressive symptoms in young adults: Potential moderators and mediators. Journal of Family Issues, 25, 761-782. http://dx.doi.org/10.1177/0192513X03258313

Walsh, C., MacMillan, H., \& Jamieson E. (2003). The relationship between parental substance abuse and child maltreatment: findings from the Ontario health Child Abuse and Neglect, 27(12), 1409-1425. http://dx.doi.org/10.1016/j.chiabu.2003.07.002

Wang, M., Haertel, G., \& Walberg, H. (1994). Educational resilience in inner cities. In M. Wang \& E. Gordon (Eds.), Educational resilience in inner-city America: Challenges and prospects (pp. 45-72). Hillsdale, NJ: Erlbaum.

Werner, E. E. (1990). Protective factors and individual resilience. Handbook of Early Childhood Intervention, 2, 115-132.

Werner, E. \& Smith, R. (1982). Vulnerable but invincible. New York: McGraw Hill.

Werner, E., \& Smith, R. S. (1992). Overcoming the odds: High risk children from birth to adulthood. Ithaca, NY: Cornell University Press.

Whitbeck, L. B. \& Simons, R. L. (1990). Life on the streets: The victimization of runaway and homeless adolescents. Youth and Society, 22, 108-125. http://dx.doi.org/10.1177/0044118X90022001007

White, J. L., Moffitt, T. E., \& Silva, P. A. (1989). A prospective replication of the protective effects of IQ in subjects at high risk for juvenile delinquency. Journal of Consulting and Clinical Psychology, 57(6), 719-724. http://dx.doi.org/10.1037/0022-006X.57.6.719

Wolak, J., \& Finkelhor, D. (1998). Children exposed to partner violence. In J. Jasinski \& L. Williams (Eds.), Partner violence: A comprehensive review of 20 years of research (pp. 184-209). Thousand Oaks, CA: Sage.

Wolfe, D. A., Crooks, C. V., Lee, V., McIntyre-Smith, A., \& Jaffe, P. G. (2003). The effects of children's exposure to domestic violence: A meta-analysis and critique. Clinical child and family psychology review, 6(3), 171-187. http://dx.doi.org/10.1023/A:1024910416164

Wyman, P. A., Cowen, E. L., Work, W. C., Hoyt - Meyers, L., Magnus, K. B., \& Fagen, D. B. (1999). Caregiving and developmental factors differentiating young at - risk urban children showing resilient versus stress - affected outcomes: A replication and extension. Child development, 70(3), 645-659. http://dx.doi.org/10.1111/1467-8624.00047

Yang, K. S. (1981). Social orientation and individual modernity among Chinese students in Twain. Journal of Social Psychology, 113, 159-170. http://dx.doi.org/10.1080/00224545.1981.9924368

Yu, S. (1997) Special overview: political enthusiasm exists side by side with political indifference, Chinese Education and Society, 30(3), 65-72. http://dx.doi.org/10.2753/CED1061-1932300365 


\section{Copyright Disclaimer}

Copyright reserved by the author(s).

This article is an open-access article distributed under the terms and conditions of the Creative Commons Attribution license (http://creativecommons.org/licenses/by/3.0/). 